\title{
2013 Koiter Medal Paper: Crack-Tip Fields and Toughness of Two-Dimensional
}

\section{Elasto-Plastic Lattices}

\author{
H C Tankasala, V S Deshpande and N A Fleck* \\ Cambridge University Engineering Dept., Trumpington St., Cambridge, CB2 1PZ, U.K., \\ *Corresponding author. Email: naf1@eng.cam.ac.uk
}

\begin{abstract}
The dependence of the fracture toughness of two dimensional elasto-plastic lattices upon relative density and ductility of cell wall material is obtained for four topologies: the triangular lattice, kagome lattice, diamond lattice, and the hexagonal lattice. Crack tip fields are explored, including the plastic zone size and crack opening displacement. The cell walls are treated as beams, with a material response given by the Ramberg-Osgood law. There is choice in the criterion for crack advance, and two extremes are considered: (i) the maximum local tensile strain anywhere in the lattice attains the failure strain, or (ii) the average tensile strain across the cell wall attains the failure strain (which can be identified with the necking strain). The dependence of macroscopic fracture toughness upon failure strain, strain hardening exponent and relative density are obtained for each lattice, and scaling laws are derived. The role of imperfections in degrading the fracture toughness is assessed by random movement of the nodes. The paper provides a strategy for obtaining lattices of high toughness at low density, thereby filling gaps in material property space.
\end{abstract}

Keywords: constitutive modelling of materials, mechanical properties of materials, micromechanics, plasticity

\section{Introduction}

Two dimensional (2D) lattice materials show promise for a wide range of applications ranging from structural armour to lightweight support for satellites, and are commonly used as the core of a sandwich panel $[1,2]$. Square lattices made from the elastic-brittle ceramic cordierite are used in catalytic converters and in particulate filters for automobiles, and the fracture properties of these have been explored recently [3,4]. Less is known about the fracture toughness of metallic lattices, such as titanium lattices with potential application in jet blast deflection structures and in heat exchangers [5-7]. Lattice materials offer the possibility of high strength and toughness at low density. However, in order to vector material development, there is a need to determine the sensitivity of fracture toughness to the choice of cell-wall material, topology, relative density $\bar{\rho}$, cell-size and degree 
of imperfection. In so doing, there exists the opportunity to fill gaps in material property space. This is the primary objective of the current study.

The elasto-plastic crack tip fields are explored for four topologies of 2D lattice: the triangular lattice, kagome lattice, diamond lattice, and the hexagonal lattice, as shown in Fig. 1(b)-(e). Each lattice comprises struts of length $\ell$ and thickness $t$, such that the relative density $\bar{\rho}$ is given by

$$
\bar{\rho}=A \frac{t}{\ell}
$$

with the values of $A$ listed in Table 1 [8-10].

The structural properties of these lattices are sensitive to the value of co-ordination number $Z$ for each lattice [8]. When $Z$ is less than 4 , such as $Z=3$ for the hexagonal lattice, the lattice can accommodate macroscopic staining by cell wall bending without stretching. In contrast, when the co-ordination number exceeds 4 , such as $Z=6$ for the triangular lattice, macroscopic straining necessarily involves cell wall stretching, which is a much stiffer mode of deformation than cell wall bending. The transition case is $Z=4$ and a range of macroscopic behaviours is possible. For example, the diamond lattice is compliant when it is sheared along the direction of the struts, but is a stiff, stretching structure under direct straining in the strut-direction. In contrast, the kagome lattice is an isotropic, stiff, stretching structure. This broad range in behaviours motivates the choice of these four lattices in this study: the triangular, hexagonal and kagome lattices are isotropic in-plane, whereas the diamond lattice is strongly anisotropic.

Consider a lattice made from an elastic, ideally plastic solid of cell wall modulus $E_{S}$ and yield strength $\sigma_{Y S}$. The macroscopic modulus $E$ and the macroscopic yield strength $\sigma_{Y}$ in the $x_{2}$ direction of each lattice, as defined in Fig. 1 , scale with $\bar{\rho}$ according to

$$
E=B \bar{\rho}^{b} E_{S}
$$

and

$$
\sigma_{Y}=C \bar{\rho}^{c} \sigma_{Y S}
$$

Now the exponents $b$ and $c$ equal unity for a stretching lattice, and exceed unity for a bending lattice, see [8-10]. Values for $(B, b ; C, C)$ are listed in Table 1 for the four lattices of interest, as taken from [11]. We note in passing that the diamond lattice is highly anisotropic. Its shear modulus $G$ and shear strength in the in the $x_{1}-x_{2}$ reference frame of Fig. 1 are given by

$$
G=\frac{1}{4} \bar{\rho} E_{S} \quad \text { and } \quad \tau_{Y}=\frac{1}{2} \bar{\rho} \sigma_{Y S}
$$


respectively, see $[11,12]$.

Much less is known about the fracture of lattice materials. The fracture toughness of 2D elastic-brittle lattices has been recently studied by Fleck and co-workers $[3,11,13]$ and by a number of other groups [14-16]. In contrast, only preliminary studies exist on the fracture toughness of a ductile hexagonal lattice [17], and on the reduction in strength of a ductile lattice due to the presence of short cracks [18].

In the elastic-brittle case, finite element simulations were performed on selected 2D lattices containing a long crack $[3,11,13]$. In brief, a boundary layer analysis was performed, such that the outer boundary of an edge-cracked lattice was subjected to the asymptotic displacement fields associated with a remote mode I stress intensity factor, $K$. The stress state in the lattice and the location of maximum local tensile stress $\sigma_{\max }$ near the crack tip were determined. Upon equating $\sigma_{\max }$ to the fracture strength $\sigma_{f}$, the macroscopic fracture toughness $K_{I C}$ was estimated for the four lattices of Fig. 1, see $[4,13]$. It was demonstrated that $K_{I C}$ scales with $\sigma_{f}$, the cell size $\ell$ and the relative density $\bar{\rho}$ of the lattice according to

$$
K_{I C}=D \bar{\rho}^{d} \sigma_{f} \sqrt{\ell}
$$

where $(D, d)$ are tabulated in Table. The sensitivity of fracture toughness to relative density is quantified by the exponent $d$ : the fracture toughness falls rapidly with diminishing $\bar{\rho}$ for the hexagonal lattice $(d=2)$, but less rapidly for the diamond lattice $(d=1)$, triangular lattice $(d=1)$ and kagome lattice $(d=1 / 2)$. The value $d=2$ for the lattice is consistent with the fact that its cell walls bend under general in-plane loading, whereas the struts of the triangular lattice and diamond lattice stretch, giving $d=1$, see [8] for a full discussion. The kagome lattice has an exceptionally high fracture toughness (with $d=1 / 2$ ), and this is ascribed to crack tip blunting by elastic zones of shear emanating from the crack tip, see [13]. However, the fracture toughness of the kagome lattice is sensitive to geometric imperfection: Symons and Fleck [19] and Romijn and Fleck [11] have explored the knockdown in fracture toughness due to imperfections in the form of randomly displaced nodes. They found that the kagome and diamond lattices are the most imperfection sensitive, while the triangular and hexagonal lattices are imperfection-insensitive. In the current study, the significance of imperfection is re-visited for the case of ductile lattices that can undergo large deformations prior to failure.

Scope of study

This paper is in two parts. First, the mode I crack tip field is obtained numerically for an elasto-plastic lattice of topology listed in Fig. 1. Both the perfect topology and the imperfect case (random 
misalignment of the nodes) are considered. A Ramberg-Osgood description is used for the cell wall solid, such that the strain $\varepsilon$ is related to the stress $\sigma$ in uniaxial tension by

$$
\frac{\varepsilon}{\varepsilon_{0 S}}=\frac{\sigma}{\sigma_{0 S}}+\left(\frac{\sigma}{\sigma_{0 S}}\right)^{n}
$$

in terms of the 3 material parameters $\left(\sigma_{0 S}, \varepsilon_{0 S}, n\right)$, where $\sigma_{0 S}$ is the yield strength, $\varepsilon_{0 S}$ is the yield strain and $n$ is the strain hardening exponent. The plastic zone shape and size $r_{\mathrm{P}}$, and the crack tip opening displacement $\delta$ are obtained by finite element simulation of the small scale yielding problem. And scaling laws are derived for the dependence of $r_{\mathrm{P}}$ and $\delta$ upon the magnitude of $K, n$, and $\bar{\rho}$.

Second, the fracture toughness of the ductile lattices is predicted, based on the maximum value of (i) local tensile strain (LTS) at any point in the lattice, or (ii) mean tensile strain (ATS) at any cross-section of the lattice upon averaging the axial strain over the strut thickness. For both criteria, the significance of finite strain is determined. Scaling laws are obtained for the fracture toughness as a function of relative density, topology, degree of imperfection and strain hardening exponent. Finally, a scoping study is performed to determine the potential of lattices to fill gaps in material property space: the intent is to achieve lightweight materials of high toughness.

\section{The elasto-plastic crack tip field}

\subsection{Scaling relations for plastic zone size}

The crack-tip field for each of the four lattices is determined by a boundary layer analysis, such that the outer boundary of a square mesh is subjected to the displacement field associated with the mode I stress intensity factor $K$. Consider the general case of a semi-infinite edge crack in an orthotropic plate, as shown in Fig. $1(a)$. Write the displacement field in Cartesian form as $u_{i}\left(x_{j}\right)$, and introduce the polar coordinate system $(r, \theta)$ centred on the crack tip, with the crack faces lying along $\theta= \pm \pi$. The displacement field in the elastic annulus surrounding the crack-tip plastic zone scales with $K$ according to

$$
u_{i}=\frac{K \sqrt{r}}{E} f_{i}(\theta)
$$

as given by $[20,21]$ for an orthotropic plate; the lengthy but explicit formulae for the non-dimensional functions $f_{i}(\theta)$ are not repeated here. They additionally depend upon $\bar{\rho}$ for the diamond lattice since the degree of anisotropy is dependent on relative density (recall that the ratio of direct modulus 
to shear modulus scales as $\bar{\rho}^{2}$ for this lattice). The cell wall material is described by (6). Finite element (FE) calculations are performed using the commercial FE code ABAQUS (version 6.12). A 2D FE mesh is generated, with 500 struts along each edge of the domain and each strut around the crack tip represented by 70 Euler-Bernoulli beam elements for small strain calculations and 150 Timoshenko beam elements for finite strain calculations ${ }^{1}$. Both elements are two-noded and account for both bar stretching and bending deformations. The Ramberg-Osgood description (6) of J2 deformation theory is used to describe the material response. Note that loading is close to proportional for the mode I crack tip field and so deformation theory and flow theory predictions almost coincide. (This was confirmed in the present study by performing selected calculations using J2 flow theory). A previous study on predicting the fracture toughness of brittle lattices [13] concluded that the difference in fracture toughness is negligible when the peripheral nodes of the mesh are subjected to the material rotation associated with the asymptotic $K$-field or are unconstrained in rotation. Thus, it suffices to apply only translational displacements on the boundary nodes and to allow boundary nodes to have free rotation.

As the applied $K$ is increased via the peripheral nodal displacements, a plastic zone develops at the crack tip and envelopes an increasing number of units cells. In order to define the plastic zone boundary, we make the choice that the cell wall material yields when the von Mises measure of total strain exceeds a value of $2 \varepsilon_{0 S}$. Since the plastic zone is not circular in shape, write $r_{\mathrm{P}}$ as the maximum extent of plastic zone from the crack tip, at an inclination $\omega$ to the cracking plane. Thus, $\left(r_{\mathrm{P}}, \omega\right)$ are the polar co-ordinates of the maximum radial extent of the plastic zone. Recall that the plane strain plastic zone size for a fully dense elastic, ideally plastic solid of yield strength $\sigma_{Y S}$ is given by

$$
r_{\mathrm{P}}=\frac{1}{3 \pi}\left(\frac{K}{\sigma_{Y S}}\right)^{2}
$$

and we anticipate a similar scaling for the lattices, provided that we replace $\sigma_{Y S}$ by the effective yield strength of the lattice $\sigma_{Y}$, as defined in Table 1. Consider the case of an elastic, ideally plastic lattice with $n=\infty$ in (6), such that $\sigma_{0 S}=\sigma_{Y S}$ and. $\varepsilon_{0 S}=\sigma_{Y S} / E_{S}$. Finite element simulations have been performed to determine the plastic zone shape and size as a function of $K$, and a regression analysis confirms that

$$
r_{\mathrm{P}}=\alpha_{1}\left(\frac{K}{\sigma_{Y}}\right)^{2}
$$

\footnotetext{
${ }^{1}$ Euler-Bernoulli beam elements are appropriate only for large rotations and small strains as the crosssectional thickness change is ignored. Timoshenko beam elements use a fully nonlinear formulation so that the strains and rotations can be arbitrarily large.
} 
for each of the triangular, hexagonal and kagome lattices. (A different scaling applies to the diamond lattice, see below.) The best fit values of $\alpha_{1}$ and are given in Table 2 as a function of $n$ for each lattice; the inclination $\omega$ to the cracking plane, associated with the maximum radial extent of plastic zone, is almost independent of $n$ and is included in Table 2 as a single entry for each lattice. We can compare the predictions of $\alpha_{1}$ for the hexagonal lattice with the previous estimate by [17] for a lattice made from a solid of bilinear stress-strain law. The numerical simulations as given in Fig. 5 of [17] agree with their analytical estimate (14), and they obtain $\alpha_{1}=0.32$ at $\omega=71^{\circ}$; this is in good agreement with the value of $\alpha_{1}$ as reported here of 0.17 to 0.36 depending upon the choice of $n$.

The diamond lattice does not obey the scaling (9): a regression analysis (not plotted here for the sake of brevity) reveals that

$$
r_{\mathrm{P}}=\alpha_{1} \bar{\rho}\left(\frac{K}{\sigma_{Y}}\right)^{2}
$$

This is a consequence of the fact that its shear strength is much less than its axial strength (in axes aligned with the lattice). The redefined values of $\alpha_{1}$ are included in Table 2.

A plot of the plastic zone for each lattice (with $n=\infty$ ) is given in Fig. 2 for the converged case where the plastic zone envelopes many unit cells (so that the lattice behaves as an effective medium). The axes $\left(x_{1}, x_{2}\right)$ have been normalised with regard to the value of $r_{\mathrm{P}}$ for each lattice. The orientation $\omega$ along which the plastic zone has maximum extent is indicated in Fig. 2 by a solid line emanating from the crack tip to the plastic zone boundary. There is marked difference in plastic zone shape from lattice to lattice, with the triangular lattice closest to that of a fully dense solid (see for example Fig. 2.36 of [22]). The plastic zone of each lattice has two lobes, a primary one pointing forwards and a smaller one pointing backwards. The difference between the triangular and kagome lattice is striking since both are isotropic, stretching lattices in the elastic state. We note that the orientation $\omega$ along which the plastic zone has maximum extent is close to the strut orientation for all lattices. The plastic zone extends only a small distance directly ahead of the crack tip for the diamond and hexagonal lattices (see Fig. 2(c) and (d), respectively) and this can be traced to the fact that the stress state directly ahead of the crack tip is close to hydrostatic, and these lattices have a much greater hydrostatic strength than shear strength. Further, the extreme anisotropy of the diamond lattice, with a low shear strength along the strut directions leads to two elongated lobes at $\omega= \pm 45^{\circ}$. There is only a minor effect of strain hardening index $n$ upon the shape and size of the plastic zone, consistent with the case of a fully dense solid, see for example Fig. 2.36 of [22]. To give direct evidence for this, the plastic zones for $n=3$ and 10 are included in Fig. 2, with axes still normalised by the value of $r_{\mathrm{P}}$ for $n=\infty$. For each lattice the plastic zone shrinks slightly with decreasing $n$. 


\subsection{Scaling relations for crack opening displacement}

Consider the crack opening profile $\delta(r)$ as a function of distance $r$ from the crack tip for each lattice. A typical crack opening profile for the elasto-plastic lattices, with $n=10$, is given in Fig. 3 . The dependence of $\delta$ upon $(r, K, \bar{\rho}, n)$ has already been given in [13] for the linear case, $n=1$. Similar scaling arguments apply to the non-linear case, as follows. Recall from [13] that the crack tip opening profiles for linear elastic, hexagonal and triangular lattices are adequately approximated by the crack tip solution for an elastic continuum down to $r$ on the order of $\ell$. Consequently, we anticipate that the crack opening profile for the elasto-plastic hexagonal and triangular lattices can be represented by the opening profile for a dilatant elasto-plastic solid. Now, Pan and co-workers [23] have shown that the asymptotic form of the Hutchinson-Rice-Rosengren (HRR) solution for the crack opening profile $\delta(r)$ is maintained in the compressible case, such that

$$
\delta(r)=\alpha_{2} \varepsilon_{0} r\left(\frac{K}{\sigma_{0} \sqrt{r}}\right)^{\frac{2 n}{n+1}}
$$

where $\alpha_{2}$ is dependent upon $n$. We have explored the ability of (11) to describe the crack tip opening of the lattice as a function of $(r, K, \bar{\rho}, n)$. Note that the formula (11) makes use of the effective properties of each lattice $\sigma_{0}=\sigma_{Y}, E$, and $\varepsilon_{0}=\varepsilon_{Y}=\sigma_{Y} / E$, and these are related to the cell wall properties by making use of formulae (1-3) along with the coefficients listed in Table 2. A good fit is obtained but is not shown here for the sake of space. The reader is referred to [13] for a full discussion for the linear case; non-linearity does not change the conclusions but does modify the best fitting values for $\alpha_{2}$ for the hexagonal and triangular lattices, as given in Table 2.

Next, consider the kagome lattice. For the linear case, $n=1$, it was observed in [13] that an elastic blunting phenomenon occurs and the crack opening profile of the kagome lattice exceeds that of the continuum solution for an isotropic elastic solid for $r / \ell<20$. Now limit attention to the crack tip opening $\delta$ at one joint back from the crack tip. Consequently, some modification to the formula (11) is needed for the kagome lattice. Numerical simulations reveal that

$$
\delta=\alpha_{2} \varepsilon_{0} \ell\left(\frac{K}{\sigma_{0} \sqrt{\ell}}\right)^{\frac{2 n}{n+1}} \bar{\rho}-\left(\frac{1}{n+1}\right)
$$

to a good approximation (not shown). A similar phenomenon of crack tip blunting is observed for the diamond lattice, as discussed in [11] for the linear case. The crack tip opening one joint back from the crack tip is given by 


$$
\delta=\alpha_{2} \varepsilon_{0} \ell\left(\frac{K}{\sigma_{0} \sqrt{\ell}}\right)^{\frac{2 n}{n+1}} \bar{\rho}
$$

for the diamond lattice. The values of $\alpha_{2}$ are summarised in Table 2 for the kagome and diamond lattices, for $n$ in the range of 1 to $\infty^{2}$. We will account for the differences in behaviour (11)-(13) in a subsequent section of this paper.

\section{The predicted fracture toughness}

The numerical simulations of the crack tip field in each lattice are used to estimate the initiation value of fracture toughness, $K_{I C}$. We emphasise that there is choice in the local fracture criterion. Fleck and co-workers previously analysed an elastic-brittle lattice and used a local maximum tensile stress criterion. Here, we focus on ductile lattices, and we make use of a local strain criterion. Beam elements are adopted, with a linear distribution of strain across the thickness, with an average value $\varepsilon_{A}$ and a maximum tensile value $\varepsilon_{T}$ on the outermost fibre. Two criteria are considered:

(i) the maximum local tensile strain $\varepsilon_{T}$ anywhere in the lattice attains the failure strain termed the Local Tensile Strain (LTS) criterion, or

(ii) the mean tensile strain $\varepsilon_{A}$ across the cell wall attains the failure strain (which can be identified with the Considere necking strain or a smaller strain if some form of damage intervenes), termed the Average Tensile Strain (ATS) criterion.

We shall employ both criteria for completeness. The ATS criterion is most pertinent to highly ductile solids that fail by necking in the presence of some bending, as discussed by [24]. In contrast, brittle alloys such as high strength aluminium alloys can develop cracks (for example by shear localisation) when a maximum tensile strain is achieved, and the LTS criterion applies, see for example [25-27]. We anticipate that the hexagonal lattice is bending-dominated such that $\varepsilon_{A} \ll \varepsilon_{T}$ whereas the other three lattices are stretching-dominated with the feature that $\varepsilon_{A} \approx \varepsilon_{T}$.

\section{(i) Predictions according to the LTS criterion}

Predictions for the maximum value of $\varepsilon_{T}$ versus $K$ in the crack tip plastic zone are plotted in Fig.4 (a)-(d) for the four lattices. Both small strain and finite strain analyses are considered, and results are presented for the choice $n=10$ and $\varepsilon_{0 S}=0.001$, and for selected values of $t / \ell$. (Additional

\footnotetext{
${ }^{2}$ We note in passing that the formula (12) is slightly different from the expression reported in [13] for the case $n=1$ and we ascribe the slight difference to the more refined numerical simulations performed herein.
} 
simulations were performed for $n=3$ and $n=\infty$, and results are listed in Table 3 for fracture toughness predictions, but are omitted from the plots for the sake of brevity.) For each topology, the response is elastic and $\varepsilon_{T}$ scales linearly with $K$ in the regime $\varepsilon_{T}<\varepsilon_{0 S}$. At larger values of $\varepsilon_{T}$ a crack tip plastic zone is present, and the magnitude of $\varepsilon_{T}$ increases with $K$ in a power law manner. Since the singular field for a compressible power-law plastic solid is of HRR type, we deduce that

$$
\varepsilon_{T} \propto K^{\frac{2 n}{n+1}}
$$

for the small strain solution. Numerical checks (not shown here) have been performed to confirm this for $n$ in the range 1 to 500 (which we treat as the elastic, ideally-plastic limit). We note from Fig. 4 that the finite strain solution for $\varepsilon_{T}$ exceeds the small strain solution by upon to a factor of 2 at large values of $K$. A regression fit to the curves of Fig. 4 for the small strain and finite strain simulations has been performed beyond yield, and gives for each lattice

$$
K=D \bar{\rho}^{d}\left(\frac{\varepsilon_{T}}{\varepsilon_{0 S}}\right)^{\frac{n+1}{2 n}} \sigma_{0 S} \sqrt{\ell}
$$

upon making use of (5). The deduced values for $(D, d)$ are listed in Table 3 for both the small and finite strain assumptions. We note in passing that $D$ is remarkably insensitive to the value of $n$ except for the hexagonal lattice - the only bending-dominated lattice under consideration.

The sensitivity of the $K$ versus $\varepsilon_{T}$ relation to the lattice topology at $\bar{\rho}=5 \%$ is given in Fig. $5(a)$, for both the small and finite strain cases. Upon assuming the LTS criterion of crack extension the kagome lattice has the highest toughness, followed by the triangular, diamond and hexagonal lattice.

\section{(ii) Predictions according to the ATS criterion}

In order to assess whether this ranking as given in Fig. 5(a) for the LTS criterion also applies for the ATS criterion, we plot in Fig. $5(b)$ the $K$ versus $\varepsilon_{A}$ relation for the four lattices at $\bar{\rho}=5 \%$. The ranking is maintained in the finite strain simulations. Further, the choice of small versus finite strain assumption has only a minor effect upon the $K$ versus $\varepsilon_{A}$ relation for the kagome, triangular and diamond lattices. In contrast, the $K$ versus $\varepsilon_{A}$ response of the hexagonal lattice is sensitive to the choice of small versus finite strain: the small strain assumption does not capture the stretching that develops with finite rotation of the struts near the crack tip. The progressive alignment in the direction of loading leads to a switch from bending to stretching, and this is captured by the finite 
strain simulations but not the small strain simulations, see the deformed meshes for both types of simulation in the insert of Fig. 5(b).

It is instructive to compare the LTS and ATS criteria for finite strain of the four lattices in Fig. 5(c), again for $\bar{\rho}=5 \%$. There is small effect of choice of failure criterion upon the predicted toughness for the kagome, triangular and diamond lattice, with a slightly tougher behaviour upon assuming the ATS criterion. In contrast, the toughness of the hexagonal lattice is highly sensitive to the choice of ATS versus LTS criterion. The ATS criterion implies a fracture toughness which increases only slightly with increasing value of $\varepsilon_{A}$ whereas the LTS criterion implies a lower fracture toughness that increases more rapidly with increasing value of $\varepsilon_{T}$. A power law fit of $K$ versus $\varepsilon_{A}$ for the hexagonal lattice has been conducted for $\bar{\rho}$ in the range of $1 \%$ to $10 \%$ and $n=3,10$ and $\infty$. The curves are not shown for the full range of assumed $\bar{\rho}$ and $n$ (for the sake of brevity), but are similar to the one given in Fig. 5(b) for the ATS criterion. The correlation is given by

$$
K=14 \bar{\rho}^{2}\left(\frac{\varepsilon_{A}}{\varepsilon_{0 S}}\right)^{\frac{1}{n}} \sigma_{0 S} \sqrt{\ell}
$$

in contrast to (15) for the LTS criterion. We further note from Fig. 5(c) that, with increasing magnitude of failure strain (from the yield value of 0.001 to the necking strain of 0.1 ), the values of $K$ by the two criteria converge for the hexagonal lattice. The sensitivity of toughness of the hexagonal lattice to the failure criterion is striking, but awaits experimental validation.

\section{Analytical models for the fracture toughness of each lattice}

Assume that the fracture toughness of an elasto-plastic lattice is dictated by the failure of the most highly strained cell wall at the crack tip. In the approximate treatment of the present section, we assume that stretching-dominated lattice such as the triangular lattice fail when the average value of axial strain over the cross-section $\varepsilon_{A}$ achieves a failure value $\varepsilon_{f}$, whereas a bending-dominated lattice such as the hexagonal lattice fails when the bending strain at the outer fibre $\varepsilon_{B}$ achieves a failure value $\varepsilon_{f}$. This approach extends that of $[4,11,13]$ who considered elastic-brittle lattices and assumes that the toughness is dictated by a local maximum tensile stress criterion.

Consider the case of a semi-infinite crack in each lattice, with cell-wall properties given by (6), and loaded by a mode $I K$-field. In a Cartesian reference frame centred at the crack tip, as shown in Fig. 1 , the crack is aligned with the $x_{1}$ axis, and lies normal to the $x_{2}$ axis. A plastic zone exists near the crack tip, and as the crack tip is approached the effective, macroscopic stress $\sigma_{i j}$ and macroscopic 
strain $\varepsilon_{i j}$ will approach that of $J$-field for a dilatant plastic solid. This asymptotic field is a variant of the well-known HRR field for an isotropic von Mises solid with strain hardening. In particular, the tensile macroscopic stress $\sigma_{22}$ at a distance $x_{1}$ ahead of the crack tip, and the tensile macroscopic strain $\varepsilon_{22}$ scale with the J-integral according to

$$
J \approx \sigma_{22} \varepsilon_{22} x_{1}
$$

as discussed by $[28,29]$. The relationship between macroscopic stress and cell-wall stress (and likewise between macroscopic strain and cell-wall strain) is dependent upon the lattice topology. Consider each case in turn.

\subsection{Triangular lattice and diamond lattice}

The triangular lattice is stretching-dominated, and so the cell wall at any location is subjected to an axial tensile stress $\sigma_{S}$ and to tensile strain $\varepsilon_{S}$. Directly ahead of the crack tip, the normal traction $\sigma_{22}$ is related to $\sigma_{S}$ via $\sigma_{22}=\sigma_{S} \bar{\rho} / 3$ (recall the relationship (3) between the macroscopic stress and cell wall stress as given in Table 1 ) whereas the macroscopic and cell wall strains scale as $\varepsilon_{22} \approx \varepsilon_{S}$, see for example [8]. Near the crack tip, the power law term in (6) dominates the linear term, and (17) gives

$$
J \approx \frac{1}{3}\left(\frac{\varepsilon_{S}}{\varepsilon_{0 S}}\right)^{\frac{n+1}{n}} \sigma_{0 S} \varepsilon_{0 S} \bar{\rho} x_{1}
$$

Now invoke a fracture criterion: assume that the toughness $J_{I C}$ is obtained by equating $\varepsilon_{S}$ to the tensile ductility $\varepsilon_{f}$ within a cell wall at a critical distance $x_{1}=\ell$ ahead of the crack tip, giving

$$
J_{I C} \approx \frac{1}{3}\left(\frac{\varepsilon_{f}}{\varepsilon_{0 S}}\right)^{\frac{n+1}{n}} \sigma_{0 S} \varepsilon_{0 S} \bar{\rho} x_{1}
$$

The fracture toughness $K_{I C}$ is related to $J_{I C}$ in the usual manner, $K_{I C}=\sqrt{E J_{I C}}$ where the macroscopic modulus $E=E_{S} \bar{\rho} / 3=\sigma_{0 S} \bar{\rho} /\left(3 \varepsilon_{0 S}\right)$ has already been given in (2) and Table 1. Consequently, the fracture toughness $K_{I C}$ is given by (15) where $d=1$ (as for the elastic-brittle lattice) and $D=1 / 3$ for all $n$. A regression fit to the predicted toughness is in good agreement, with $d=1$ and $D=0.45-0.52$, see Table 3. FE simulations of the crack tip elasto-plastic field reveal that the diamond lattice also behaves in a stretching manner. The relation (15) again holds, but FE simulations give $d=1$ and $D=0.14-0.22$. 


\subsection{Hexagonal lattice}

The hexagonal lattice is bending-dominated, and so we shall assume that crack advance initiates when the bending strain at the outer fibre of the cell wall $\varepsilon_{B}$ attains the failure value $\varepsilon_{f}$. The toughness is again given by (17), but we need new relations for the relationship between macroscopic stress (and strain) and cell wall values. To proceed, recall that the relationship between macroscopic yield strength and cell wall yield strength is given by (3), with the geometric constants as summarised in Table 1. This same relationship provides the connection between $\sigma_{22}$ and the tensile stress on the outermost fibre of the cell wall $\sigma_{S}$,

$$
\sigma_{22}=\frac{1}{2} \bar{\rho}^{2} \sigma_{S}
$$

Likewise, the relationship between $\varepsilon_{22}$ and $\varepsilon_{B}$ for the most highly strained section of the hexagonal lattice under remote tension reads

$$
\varepsilon_{22} \approx \frac{1}{\bar{\rho}} \varepsilon_{B}
$$

Now substitute (20) and (21) into (17), and make use of (6) to obtain a relation of the form (15) where $D=\sqrt{3} / 2=0.87$ and $d=2$. The FE analysis gives $d=2$ and $D$ in the range of 0.24 to 0.90 , recall Table 3.

\subsection{Kagome lattice}

The kagome lattice is also stretching-dominated, but has an anomalously high toughness due to the presence of shear bands at the crack tip. These shear bands involve cell wall bending and this reduces the level of tensile strain within the cell walls, as follows. Write $\delta$ as the crack tip opening displacement. The cell walls within a shear band of thickness $\ell$ undergo bending, such that the bending strain at the outermost fibre is of order

$$
\varepsilon_{f}=\varepsilon_{B} \approx \frac{t \delta}{\ell^{2}}
$$

following the argument of [4], based on Fig. 2 of that paper. The toughness is related to the traction at the crack tip $\sigma_{22}$ and to the crack tip opening displacement $\delta$ according to

$$
J \approx \sigma_{22} \delta
$$

To proceed, note that the $\sigma_{22}$ is related to the cell wall strength $\sigma_{S}$ in the same manner that the macroscopic yield strength is related to the cell wall strength, relation (3), giving 


$$
\sigma_{22}=\frac{1}{2} \bar{\rho} \sigma_{S}
$$

Near the crack tip, the power law term in (6) dominates the linear term, and upon substitution of (22) and (24) into (23) we obtain

$$
J_{I C} \approx \frac{\sqrt{3}}{2}\left(\frac{\varepsilon_{f}}{\varepsilon_{0 S}}\right)^{\frac{n+1}{n}} \sigma_{0 S} \varepsilon_{0 S} \bar{\rho} x_{1}
$$

Now make use of the Irwin relation $K_{I C}=\sqrt{E J_{I C}}$ and of (2) to obtain (15) where $d=1 / 2$ and $D=12^{-1 / 4} \approx 0.54$. We emphasise the remarkable result that the fracture toughness scales with $\bar{\rho}^{1 / 2}$. The FE regression gives $d=1 / 2$ and $D=0.13-0.21$ (see Table 3), in support of the above simple model.

\section{The role of imperfections}

In practice, lattice materials contain defects such as missing cell walls, cell walls of variable thickness (such as Plateau borders in foams), and spatial variations in relative density. Here, we consider randomly located nodes, as shown in Fig. 6 for each lattice. Assume that all nodes are radially displaced by a value $R$ but in random directions from node to node, in the initial unstressed configuration. What is the knock-down in fracture toughness as a function of $R / \ell$ ? We build upon the previous study [11] wherein an elastic-brittle lattice was considered for the same topologies as that addressed here. It was demonstrated in [11] that random movement of nodes gave a knockdown in fracture toughness with increasing sensitivity from hexagonal lattice, to triangular, diamond and kagome.

A series of up to 10 structural realisations has been generated for each ductile lattice, with $n=10$ and $\varepsilon_{f}=20 \varepsilon_{0 S}$. We assume small strain behaviour, with fracture dictated by the maximum local tensile strain achieved at any location within the imperfect lattice. Write $\bar{K}_{I C}$ as the mean value of fracture toughness of the imperfect lattice over the 10 realisations, normalised by the fracture toughness for the perfect lattice of equal relative density. Then, we plot $\bar{K}_{I C}$ versus imperfection $R / \ell$ in Fig. 7 for the four lattices of relative density $\bar{\rho}=0.025$, and include the elastic-brittle result for comparison ${ }^{3}$. We note that the imperfection sensitivity of the ductile triangular lattice is comparable to that of the elastic-brittle triangular lattice; for the other three lattices the elastic-brittle case is the more imperfection-sensitive. The kagome lattice is the most imperfection-sensitive topology: the random

\footnotetext{
${ }^{3}$ In agreement with [11], we find some scatter in predicted toughness from realisation to realisation, but the overall sensitivity of toughness to imperfection is reduced in the non-linear, ductile case compared to the elastic, brittle case, and the scatter is not shown in Fig. 7.
} 
movement of nodes converts the lattice from stretching-dominated to bending dominated. Direct evidence of this behaviour is presented in Fig. 8: the power-law dependence of $K_{I C}$ upon $\bar{\rho}$ switches from $\bar{\rho}^{1 / 2}$ for the perfect lattice to $\bar{\rho}^{3 / 2}$ for the imperfect case.

\section{The realisation of tough lattices: filling gaps in material property space}

We draw on the inspiration of [30] to explore the potential of the ductile lattices at low density. It is instructive to plot the predictions of macroscopic fracture toughness for the four lattices in material property space. Choose as axes $K_{I C}$ and the density $\rho$, and add to this plot the wide range of engineering materials that currently exist, see Fig. $9(a)$. Now add the prediction (15) for a lattice of dimension $\ell=10 \mathrm{~mm}$, made from Ti-6Al-4V alloy of properties $\sigma_{0 S}=600 \mathrm{MPa}, \varepsilon_{0 S}=0.006$, $\varepsilon_{f}=50 \varepsilon_{0 S}$, and $n=10$. These predictions make use of the local total strain (LTS) criterion, and for all lattices but the hexagonal case, it is recalled that the predictions are hardly changed if the alternative ATS criterion were employed. Additionally, predictions are shown for the hexagonal lattice failing by the ATS criterion (16). The predicted fracture toughness of the kagome, triangular and diamond lattices fill gaps in material property space in the range for which the titanium lattices can be treated as a framework of beams: for $\rho$ less than $500 \mathrm{~kg} \mathrm{~m}^{-3}$. In contrast, the hexagonal lattice (by both failure criteria) offers limited advantage over existing 3D foams. It remains to manufacture and test these lattices to confirm these predictions. We emphasise that the fracture toughness of the lattices scales with $\sqrt{\ell}$ on dimensional grounds. Consequently, it is anticipated that lattices on the micron and nano scales are more brittle than the case considered here, $\ell=10 \mathrm{~mm}$. In contrast, the pertinent length scale for fully dense metallic alloys is on the order of microns, as set by the inclusion spacing in ductile fracture, for example.

It is also instructive to compare the predicted toughness $J_{I C}$ of the lattices with other engineering materials. Upon recalling that $J_{I C}=K_{I C}{ }^{2} / E$ for isotropic materials, with the role of the Poisson ratio taken to be negligible, we make use of relations (2) and (15) to give

$$
J_{I C}=H \bar{\rho}^{h}\left(\frac{\varepsilon_{f}}{\varepsilon_{0 S}}\right)^{\frac{n+1}{n}} \sigma_{0 S} \varepsilon_{0 S} \ell
$$

upon invoking the LTS criterion, $\varepsilon_{T}=\varepsilon_{f}$. The parameters $H \equiv D^{2} / B$ and $h \equiv 2 d-b$ are listed in Table 4, for convenience. For the hexagonal lattice, the toughness by the ATS criterion is significantly different from that by the LTS criterion and (2) with (16) combine to give

$$
J_{I C}=131 \bar{\rho}\left(\frac{\varepsilon_{f}}{\varepsilon_{0 S}}\right)^{\frac{2}{n}} \sigma_{0 S} \varepsilon_{0 S} \ell
$$


Expressions (26) and (27) are used to plot the predicted toughness of the three isotropic cases : triangular, kagome, and diamond lattice in Fig. 9(b), along with the typical values for other engineering materials. It is striking that the toughness of these lattices lay significantly above those of 3D foams and natural materials, with the kagome lattice the toughest at low density.

\section{Concluding remarks}

Our study reveals that the fracture toughness of ductile lattices is sensitive to the length scale of lattice in addition to relative density and choice of topology. The predicted fracture toughness is only mildly sensitive to the details of the failure criterion (maximum local tensile strain versus average tensile strain across the cell wall) for the triangular, kagome, and diamond lattice. In contrast, the hexagonal lattice has extreme sensitivity to the choice of local failure criterion: when the local maximum strain dictates the toughness, the lattice has a relatively low toughness (and is sensitive to its relative density), whereas the hexagonal lattice has high toughness when the average tensile strain dictates failure. We have also demonstrated that ductile lattices are less imperfection-sensitive than their elastic-brittle counterparts. We predict that gaps in material property space can be filled by the kagome lattice made from ductile alloys such as titanium alloys.

The current study is a detailed analysis of four lattices in a particular orientation. It is recognised that a rotation of the lattices will change the quantitative predictions of fracture toughness. The qualitative trends for the triangular, hexagonal and kagome lattices are insensitive to the lattice orientation, as the triangular and kagome lattices are stretching-governed in all orientations, while the hexagonal lattice is bending-governed in all orientations. These three lattices are elastically isotropic but have plastic anisotropy and this will perturb the shape and size of the plastic zone, and the associated toughness. Deshpande and Fleck [31] have shown that a $30^{\circ}$ rotation of the triangular lattice leads to a 50\% change in uniaxial tensile strength, while Hutchinson et al [32] find a factor of 2 difference in strength for the kagome lattice, and Gibson and Ashby [10] report an invariant response for the hexagonal lattice. These factors are independent of relative density. A full characterisation of the orientation-dependence of toughness for these lattices is beyond the scope of the present study. We emphasise that random imperfections as analysed in this study will make the lattices more isotropic in both plastic and toughness responses. The effect of orientation of the perfect diamond lattice upon strength and toughness is more pronounced than for the other lattices, as the lattice is stretching-dominated when the loading direction is aligned with the struts and is bending-dominated otherwise; this has been analysed by Romijn and Fleck [11] for the elastic case, and we expect similar trends for the non-linear solid (not analysed here).

Additionally, it remains to determine the tearing response of a ductile lattice material and the associated $R$-curve, both experimentally and theoretically. It is known from early studies on crack advance in metallic foams $[17,33,34]$ that a steeply rising $R$-curve exists and this is associated with a 
combination of crack bridging behind the advancing crack tip, and plastic dissipation within the plastic zone due to non-proportional loading effects. Open-cell metallic foams are 3D bendingdominated random lattices and behave in a similar manner to 2D hexagonal lattices. The dependence of $R$-curve behaviour upon lattice topology is an open research topic. 


\section{References}

[1] Davies, G. J., and Zhen, S., 1983, "Metallic Foams: their Production, Properties and Applications," J. Mater. Sci., 18, pp. 1899-1911.

[2] Ashby, M. F., Evans, A. G., Fleck, N. A., Gibson, L. J., Hutchinson, J. W., and Wadley, H. N. G., 2000, Metal Foams.

[3] Quintana Alonso, I., and Fleck, N. A., 2009, "Fracture of Brittle Lattice Materials : A Review," Major accomplishments in Composite materials and sandwich structures - An anthology of ONR sponsored research, I.M. Daniel, E.E. Gdoutos, and Y.D.S. Rajapakse, eds., Springer, pp. 799-816.

[4] Quintana Alonso, I., and Fleck, N. A., 2007, "Damage Tolerance of an Elastic-Brittle Diamond-Celled Honeycomb," Scr. Mater., 56(8), pp. 693-696.

[5] Wadley, H. N. G., 2006, "Multifunctional Periodic Cellular Metals," Philos. Trans. A. Math. Phys. Eng. Sci., 364, pp. 31-68.

[6] Evans, A. G., Hutchinson, J. W., Fleck, N. A., Ashby, M. F., and Wadley, H. N. G., 2001, “The Topological Design of Multifunctional Cellular Metals," Progress in Materials Science, pp. 309-327.

[7] Wadley, H. N. G., Fleck, N. A., and Evans, A. G., 2003, "Fabrication and Structural Performance of Periodic Cellular Metal Sandwich Structures," Compos. Sci. Technol., 63, pp. 2331-2343.

[8] Deshpande, V.S., Ashby, M. F., and Fleck, N. A., 2001, “Foam Topology Bending Versus Stretching Dominated Architectures," 49, pp. 1035-1040.

[9] Fleck, N. A., Deshpande, V. S., and Ashby, M. F., 2010, “Micro-Architectured Materials: Past, Present and Future," Proc. R. Soc. A Math. Phys. Eng. Sci., 466(May), pp. 2495-2516.

[10] Gibson, J. W., and Ashby, M. F., 1997, Cellular Solids: Structures and Properties, Cambridge University Press.

[11] Romijn, N. E. R., and Fleck, N. A., 2007, “The Fracture Toughness of Planar Lattices: Imperfection sensitivity," J. Mech. Phys. Solids, 55(12), pp. 2538-2564.

[12] Wang, A.-J., and McDowell, D. L., 2004, "In-Plane Stiffness and Yield Strength of Periodic Metal Honeycombs," J. Eng. Mater. Technol., 126(April 2004), p. 137. 
[13] Fleck, N. A., and Qiu, X., 2007, “The Damage Tolerance of Elastic-Brittle, TwoDimensional Isotropic Lattices," J. Mech. Phys. Solids, 55(3), pp. 562-588.

[14] Choi, S., and Sankar, B. V., 2005, "A Micromechanical Method to predict the Fracture Toughness of Cellular Materials,” Int. J. Solids Struct., 42, pp. 1797-1817.

[15] Lipperman, F., Ryvkin, M., and Fuchs, M. B., 2007, "Fracture Toughness of TwoDimensional Cellular Material with Periodic Microstructure," pp. 279-290.

[16] Huang, J. S., and Gibson, L. J., 1991, "Fracture Toughness of Brittle Foams," Acta Metall. Mater., 39, pp. 1627-1636.

[17] Schmidt, I., and Fleck, N. A., 2001, "Ductile Fracture of Two-Dimensional Cellular Structures," Int. J. Fract., 111, pp. 327-342.

[18] Cui, X., Xue, Z., Pei, Y., and Fang, D., 2011, "Preliminary Study on Ductile Fracture of Imperfect Lattice Materials," Int. J. Solids Struct., 48(25-26), pp. 3453-3461.

[19] Symons, D. D., and Fleck, N. A., 2008, "The Imperfection Sensitivity of Isotropic TwoDimensional Elastic Lattices," J. Appl. Mech., 75(5), p. 051011.

[20] Sih, G. C., Paris, P. C., and Irwin, G. R., 1965, "On Cracks in Rectilinearly Anisotropic Bodies," Int. J. Fract. Mech., 1(6), pp. 189-203.

[21] Williams, M. L., 1957, "On the Stress Distribution at the Base of a Stationary Crack," J. Appl. Mech., 24, pp. 109-114.

[22] Anderson, T. L., 1995, Fracture Mechanics: Fundamentals and Applications, CRC Press.

[23] Li, F. Z., and Pan, J., 1990, "Plane-Strain Crack-Tip Fields for Pressure-Sensitive Dilatant Materials," J. Appl. Mech., 57(1), pp. 40-49.

[24] De Kruijf, N. E., Peerlings, R. H. J., and Geers, M. G. D., 2009, “An Analysis of Sheet Necking under Combined Stretching and Bending," Int. J. Mater. Form., 2, pp. 845-848.

[25] Onck, P. R., Van Merkerk, R., De Hosson, J. T. M., and Schmidt, I., 2004, "Fracture of Metal Foams: In-Situ Testing and Numerical Modeling," Adv. Eng. Mater., 6(6), pp. 429-431.

[26] Mangipudi, K. R., and Onck, P. R., 2011, "Multiscale modelling of damage and failure in two-dimensional metallic foams," J. Mech. Phys. Solids, 59(7), pp. 1437-1461. 
[27] Mangipudi, K. R., and Onck, P. R., 2011, "Notch Sensitivity of Ductile Metallic Foams: A Computational Study," Acta Mater., 59(19), pp. 7356-7367.

[28] Rice, J. R., and Rosengren, G. F., 1968, "Plane Strain Deformation near a Crack Tip in a Power-law Hardening Material,” J. Mech. Phys. Solids, 16, pp. 1-12.

[29] Hutchinson, J. W., 1968, "Singular Behaviour at the End of a Tensile Crack in a Hardening Material.," J. Mech. Phys. Solids, 16, pp. 13-31.

[30] Ashby, M. F., 1989, "Overview No. 80: On the Engineering Properties of Materials," Acta Metall., 37(5), pp. 1273-1293.

[31] Deshpande, V. S., and Fleck, N. A., 2001, "Collapse of truss core sandwich beams in 3point bending," Int. J. Solids Struct., 38(36-37), pp. 6275-6305.

[32] Hutchinson, R. G., Wicks, N., Evans, A. G., Fleck, N. A., and Hutchinson, J. W., 2003, “Kagome plate structures for actuation," Int. J. Solids Struct., 40(25), pp. 6969-6980.

[33] Olurin, O. B., Fleck, N. A., and Ashby, M. F., 2000, "Deformation and Fracture of Aluminium Foams," Mater. Sci. Eng. A, 291(1-2), pp. 136-146.

[34] Chen, C., Fleck, N. A., and Lu, T. J., 2001, "The Mode I Crack Growth Resistance of Metallic Foams," 49, pp. 231-259. 


\section{Table captions}

Table 1. Coefficients for relative density, elastic modulus, and yield strength

Table 2. Coefficients for plastic zone size and crack tip opening

Table 3. Coefficients for fracture toughness

Table 4. Coefficients for toughness of isotropic lattices that fail by LTS criterion

\section{Figure captions}

Fig 1. Crack geometry and lattice topologies. (a) Coordinate reference frame for the lattice with crack, $(b)$ triangular lattice, $(c)$ kagome lattice, $(d)$ diamond lattice, $(e)$ hexagonal lattice

Fig 2. Mode I plastic zone for (a) triangular lattice, (b) kagome lattice, (c) diamond lattice, $(d)$ hexagonal lattice.

Fig 3. Crack tip opening profile for (a) triangular lattice, (b) kagome lattice, (c) diamond lattice, (d) hexagonal lattice.

Fig 4. Maximum value of strain in the lattice cell wall for $(a)$ triangular lattice, $(b)$ kagome lattice, $(c)$ diamond lattice, $(d)$ hexagonal lattice.

Fig 5. Mode I fracture toughness of the four topologies for $\bar{\rho}=0.05$ and $n=10$ according to (a) local tensile strain (LTS) criterion, (b) average tensile strain (ATS) criterion. (c) A comparison of predictions by the LTS and ATS criteria for the finite strain case.

Fig 6. Imperfect lattice topologies $(R / \ell=0.5)$ for $(a)$ triangular lattice, $(b)$ kagome lattice, $(c)$ diamond lattice, $(d)$ hexagonal lattice.

Fig 7. The normalised fracture toughness versus $R / \ell$ of imperfect lattices, for the choice $\bar{\rho}=0.025$ and $n=10$.

Fig 8. Dependence of fracture toughness of kagome lattice upon relative density $\bar{\rho}$ for the $R / \ell=0,0.3$ and 0.5 .

Fig 9. Material property charts (Material Property CES Selector software by Granta Design) for (a) fracture toughness versus density, (b) toughness versus density. Predictions are included for Ti-6Al-4V lattices of cell length $\ell=10 \mathrm{~mm}$ 
Table 1. Coefficients for relative density, elastic modulus, and yield strength

\begin{tabular}{lccccc}
\hline & $A$ & $B$ & $b$ & $C$ & $c$ \\
\hline Triangular & $2 \sqrt{3}$ & $1 / 3$ & 1 & $1 / 3$ & 1 \\
Kagome & $\sqrt{3}$ & $1 / 3$ & 1 & $1 / 2$ & 1 \\
Diamond & 2 & $1 / 4$ & 3 & $1 / 4$ & 2 \\
Hexagonal & $2 / \sqrt{3}$ & $3 / 2$ & 3 & $1 / 2$ & 2 \\
\hline
\end{tabular}

Table 2. Coefficients for plastic zone size and crack tip opening

\begin{tabular}{llllllllll}
\hline & \multicolumn{3}{c}{$\alpha_{1}$} & $\omega$ & \multicolumn{3}{c}{$\alpha_{2}$} \\
\hline & $n=3$ & $n=10$ & $n=\infty$ & & $n=1$ & $n=3$ & $n=10$ & $n=\infty$ \\
Triangular & 0.16 & 0.19 & 0.29 & $37^{\circ}$ & 2.73 & 1.46 & 0.97 & 0.6 \\
Kagome & 0.97 & 1.08 & 1.55 & $61^{\circ}$ & 2.73 & 2.07 & 1.73 & 1.73 \\
Diamond & 0.10 & 0.13 & 0.23 & $45^{\circ}$ & 0.67 & 0.25 & 0.07 & 0.04 \\
Hexagonal & 0.17 & 0.19 & 0.36 & $71^{\circ}$ & 13.2 & 2.54 & 0.76 & 0.52 \\
\hline
\end{tabular}

Table 3. Coefficients for fracture toughness

\begin{tabular}{lllllllll}
\hline & \multicolumn{8}{c}{$D$} \\
& $n=1$ & \multicolumn{2}{c}{$n=3$} & \multicolumn{2}{c}{$n=10$} & \multicolumn{2}{c}{$n=\infty$} & $d$ \\
\hline & & small & finite & small & finite & small & finite & \\
Triangular & 0.52 & 0.51 & 0.43 & 0.50 & 0.42 & 0.45 & 0.38 & 1 \\
Kagome & 0.21 & 0.21 & 0.17 & 0.20 & 0.16 & 0.13 & 0.09 & 0.5 \\
Diamond & 0.22 & 0.21 & 0.20 & 0.19 & 0.17 & 0.14 & 0.11 & 1 \\
Hexagonal & 0.90 & 0.76 & 0.73 & 0.52 & 0.50 & 0.24 & 0.22 & 2 \\
\hline
\end{tabular}

Table 4. Coefficients for toughness of isotropic lattices that fail by LTS criterion

\begin{tabular}{|c|c|c|c|c|c|c|c|c|}
\hline & \multicolumn{7}{|c|}{$H$} & \multirow[t]{4}{*}{$h$} \\
\hline & \multirow[t]{3}{*}{$n=1$} & \multicolumn{2}{|c|}{$n=3$} & \multicolumn{2}{|c|}{$n=10$} & \multicolumn{2}{|c|}{$n=\infty$} & \\
\hline & & small & finite & small & finite & small & finite & \\
\hline & & strain & strain & strain & strain & strain & strain & \\
\hline Triangular & 2.43 & 2.34 & 1.66 & 2.25 & 1.58 & 1.82 & 1.29 & 1 \\
\hline Kagome & 0.39 & 0.39 & 0.26 & 0.36 & 0.23 & 0.15 & 0.07 & 0 \\
\hline Hexagonal & 0.54 & 0.39 & 0.35 & 0.18 & 0.17 & 0.04 & 0.03 & 1 \\
\hline
\end{tabular}


(a)

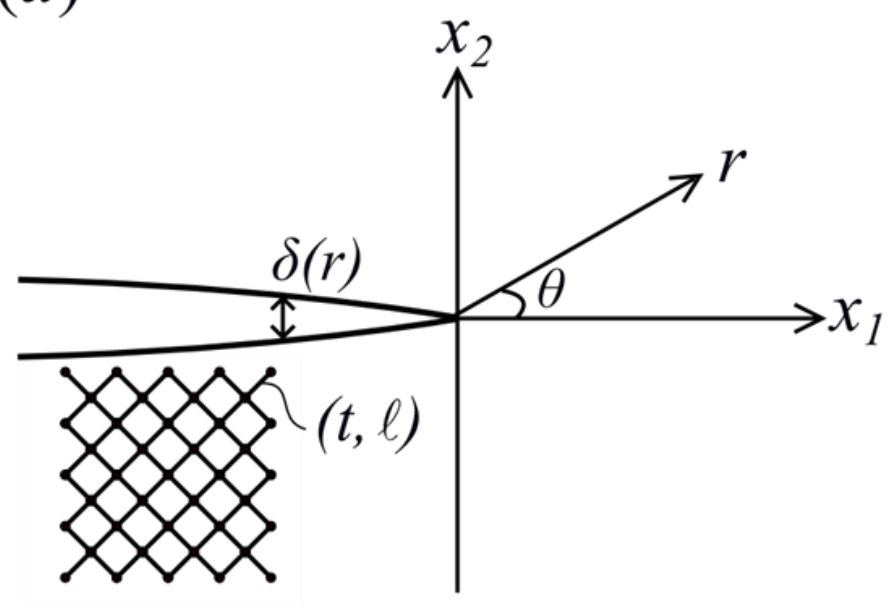

(b)

(c)
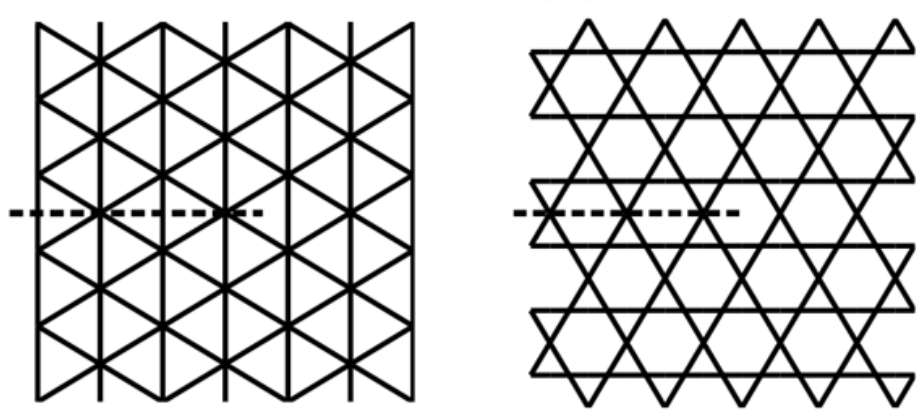

(d)

(e)

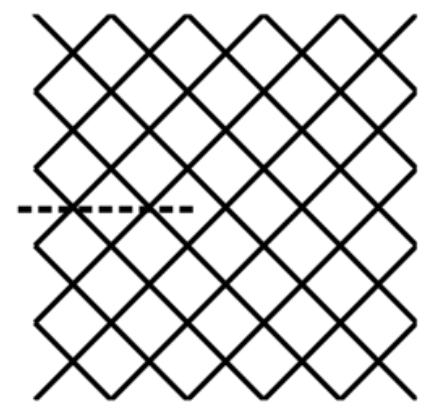

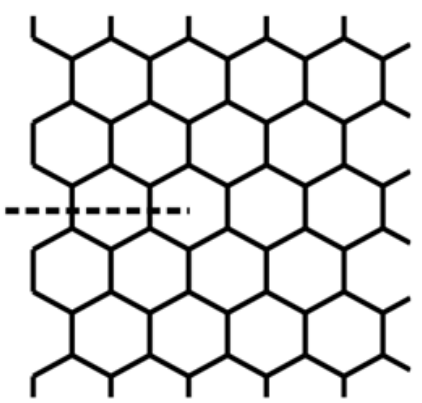

Fig 1. Crack geometry and lattice topologies. (a) Coordinate reference frame for the lattice with crack, (b) triangular lattice, (c) kagome lattice, (d) diamond lattice, (e) hexagonal lattice 
(a)

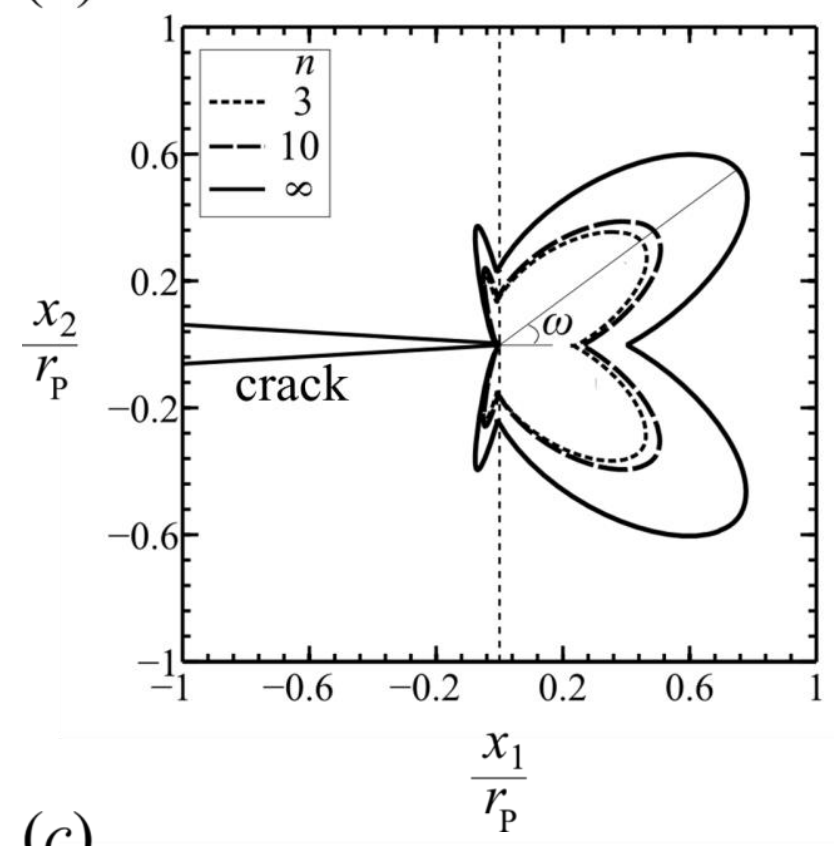

(c)

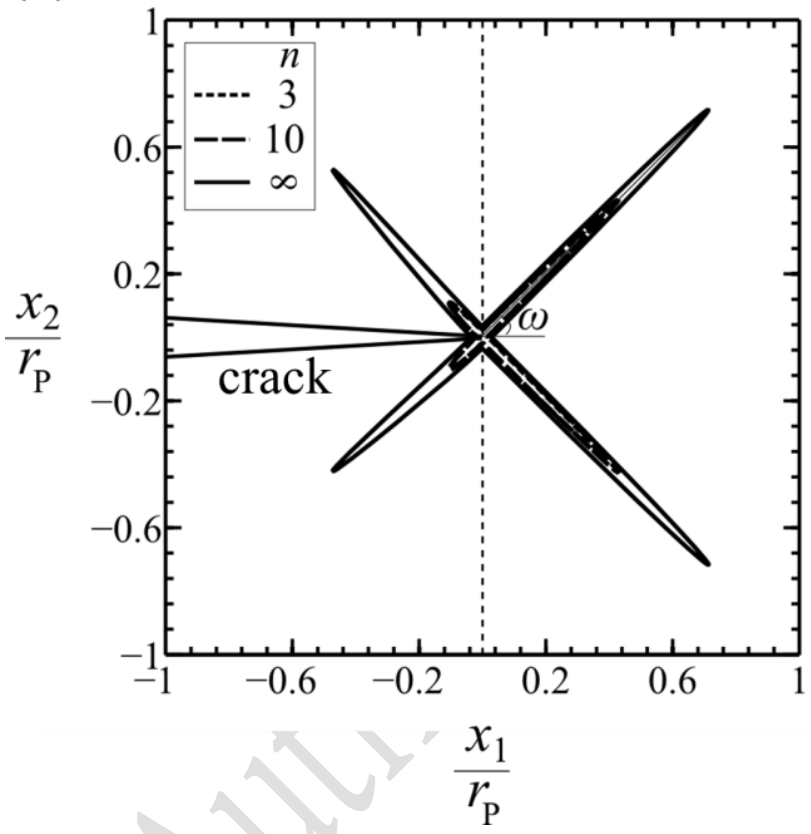

(b)

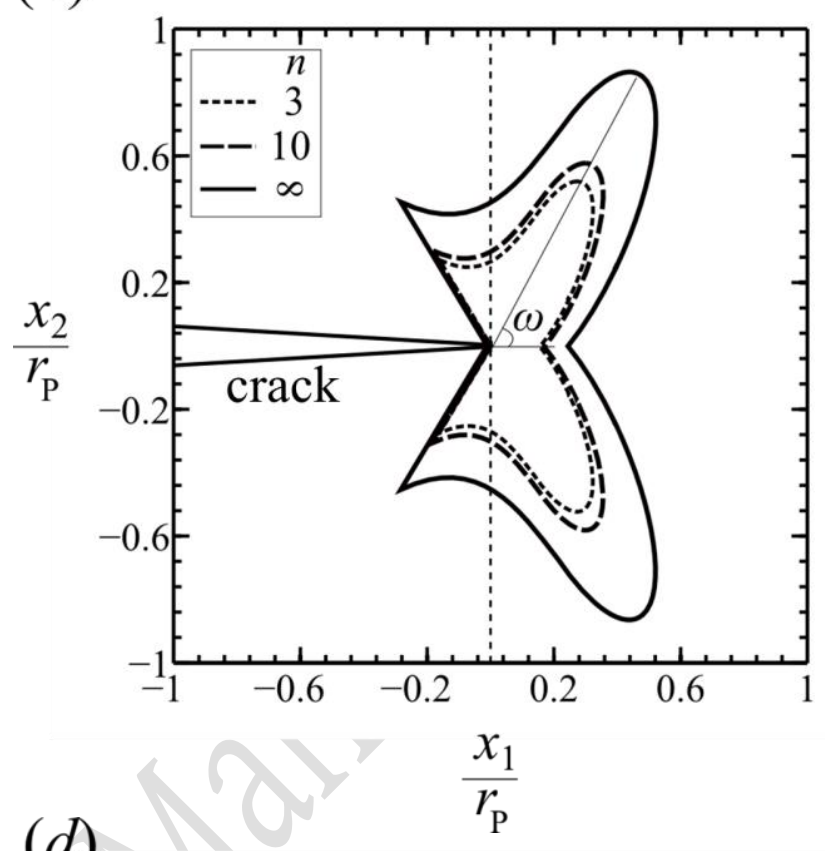

(d)

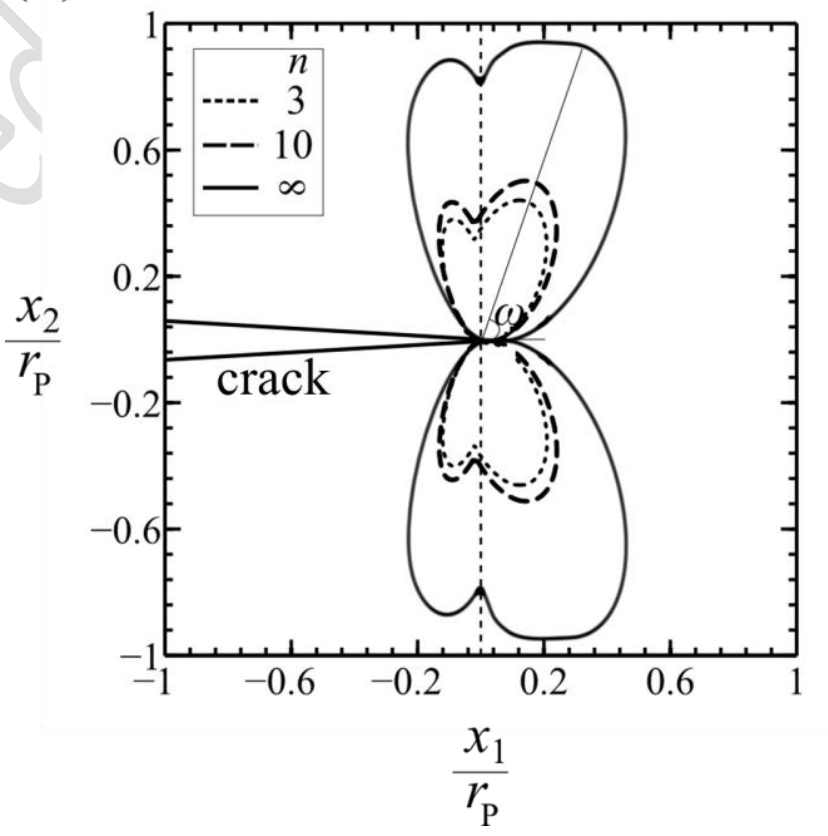

Fig 2. Mode I plastic zone for (a) triangular lattice, (b) kagome lattice, (c) diamond lattice, (d) hexagonal lattice. 
(a)

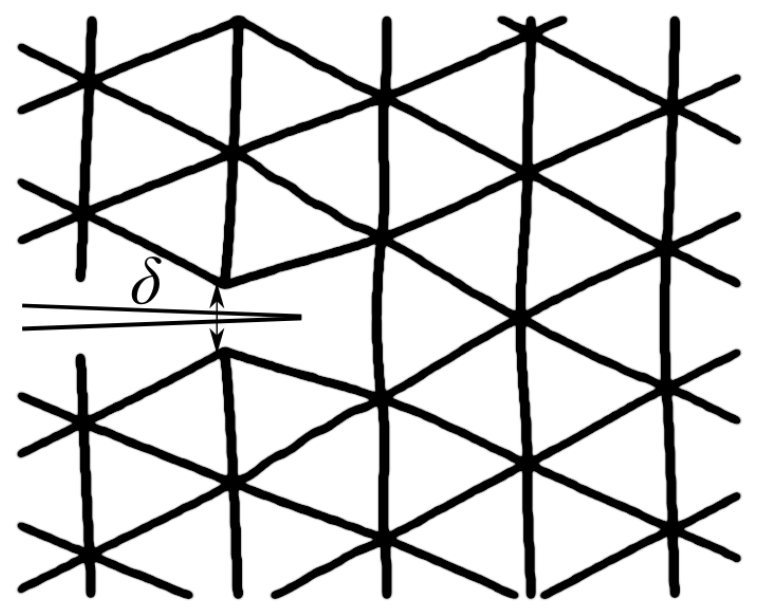

(c)

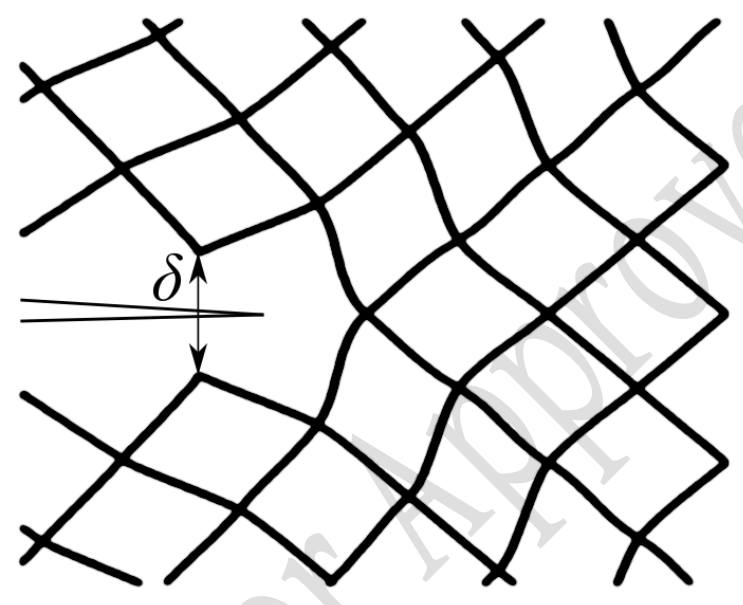

(b)

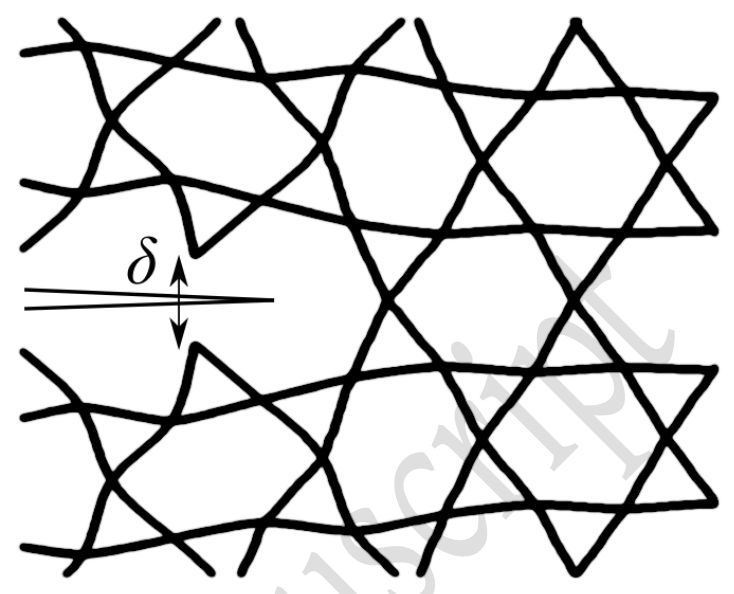

(d)

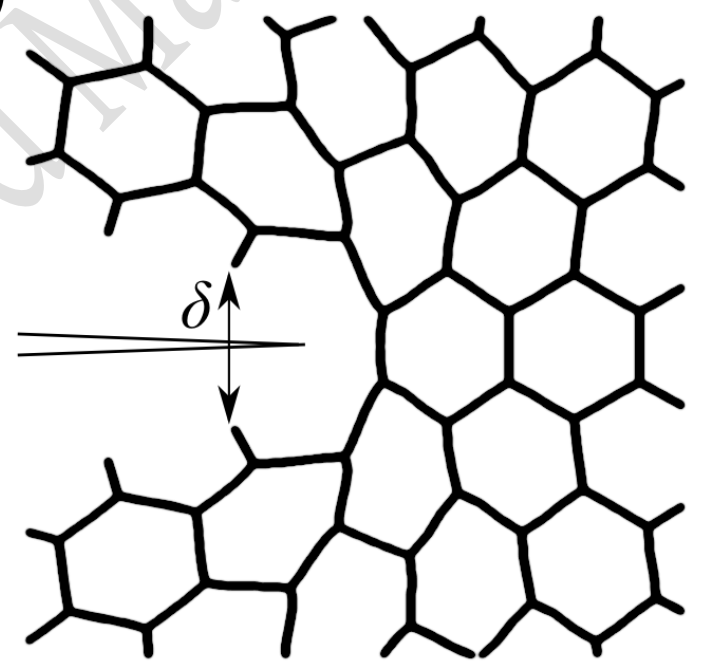

Fig 3. Crack tip opening profile for (a) triangular lattice, (b) kagome lattice, (c) diamond lattice, (d) hexagonal lattice. 
(a)

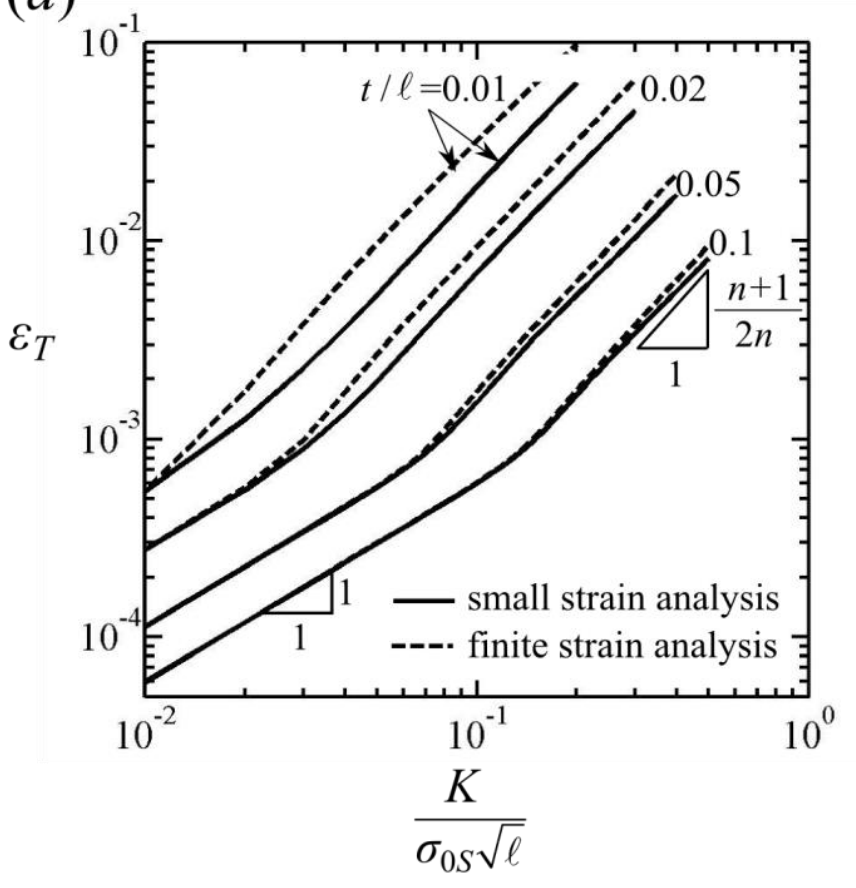

(c)

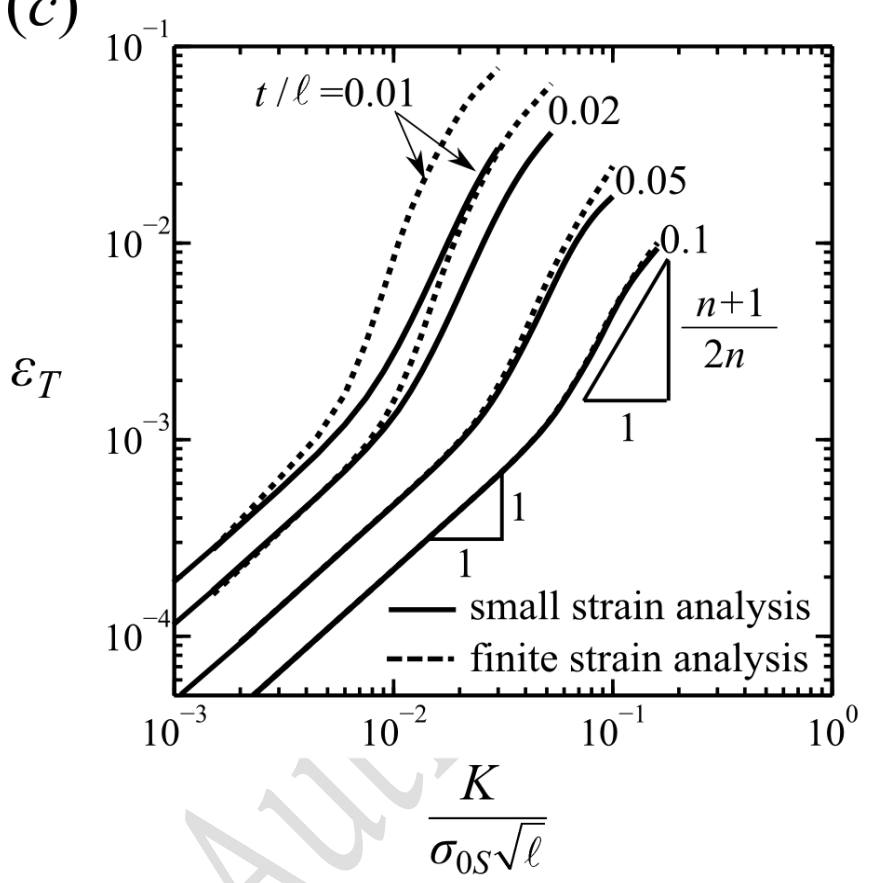

(b)

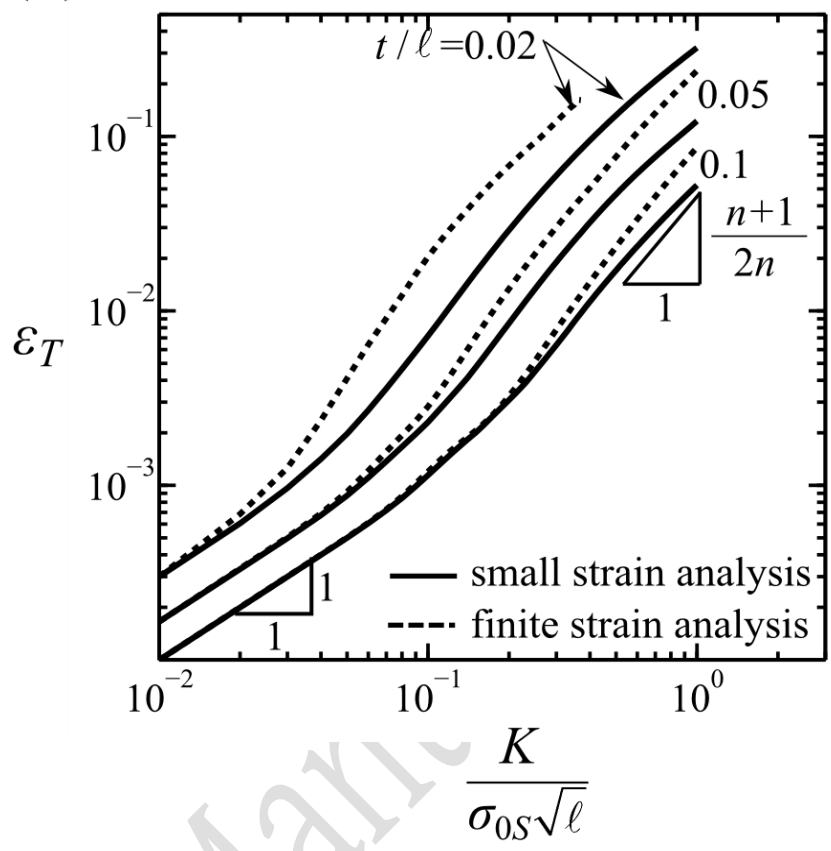

(d)

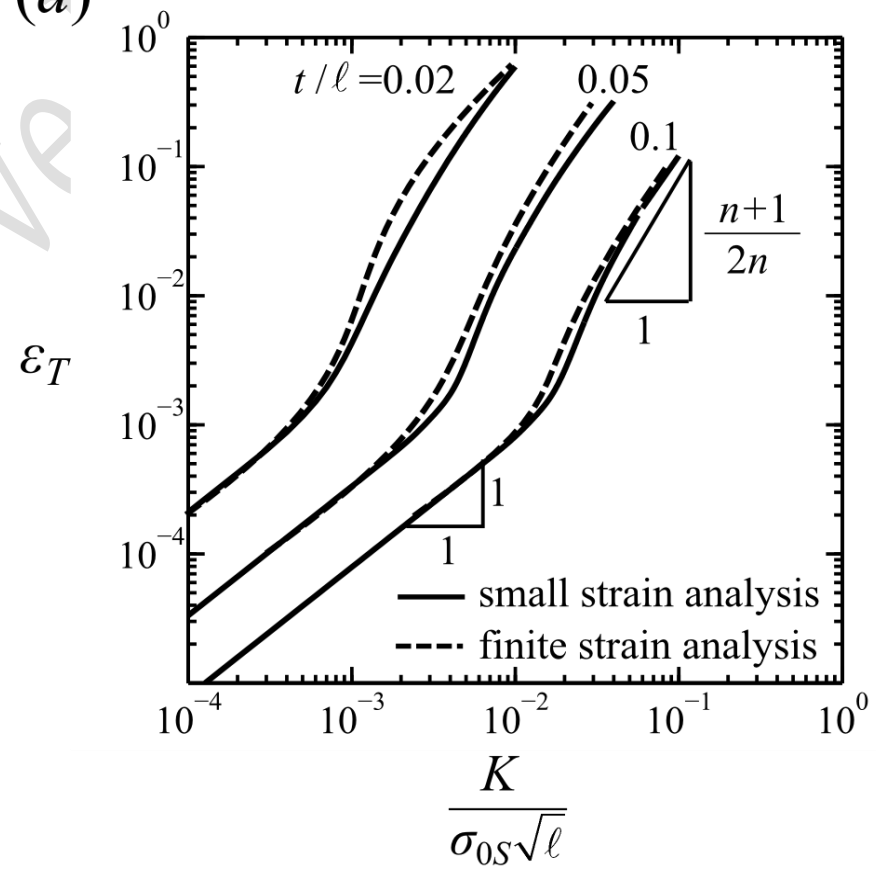

Fig 4. Maximum value of strain in the lattice cell wall for $(a)$ triangular lattice, $(b)$ kagome lattice, $(c)$ diamond lattice, $(d)$ hexagonal lattice. 


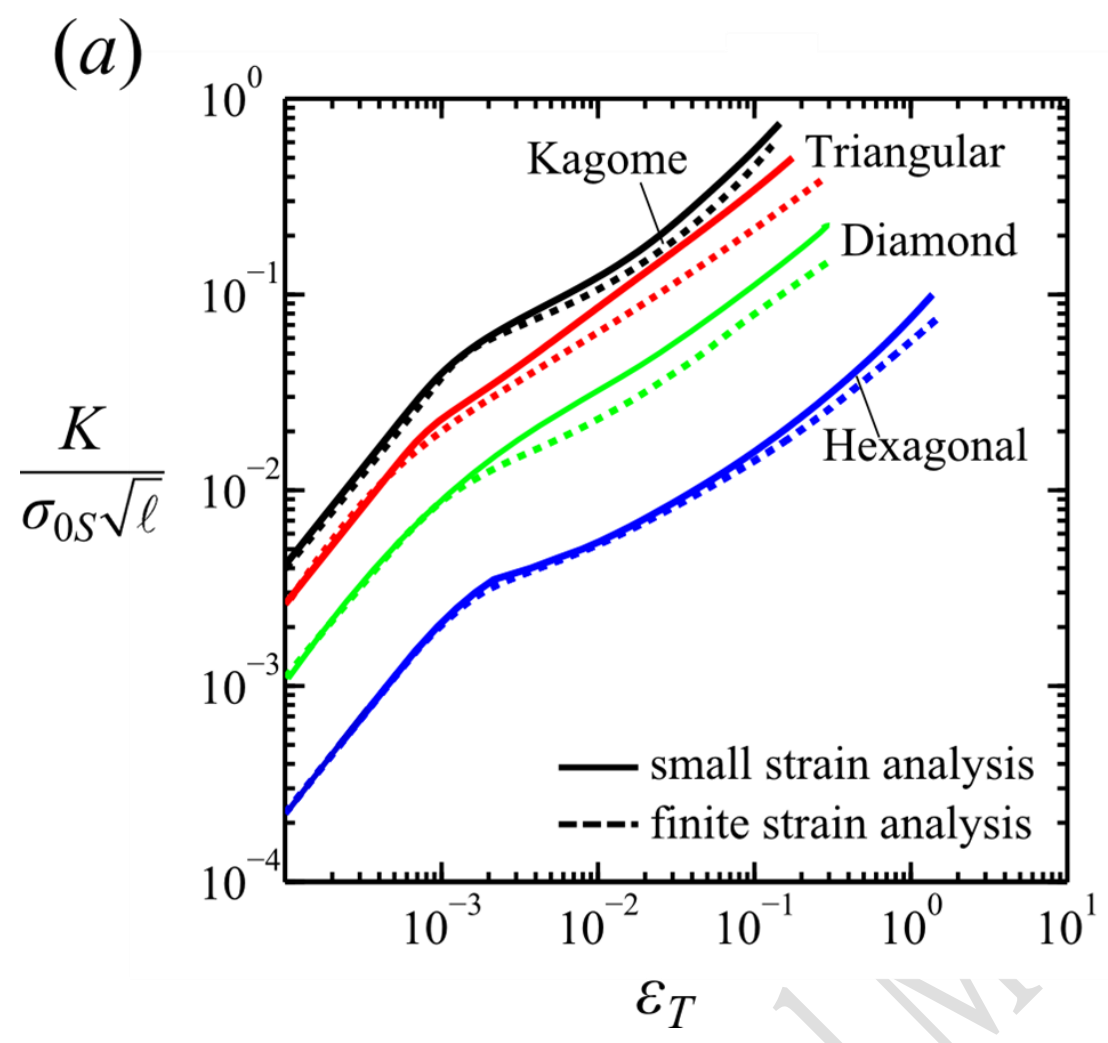

(b)

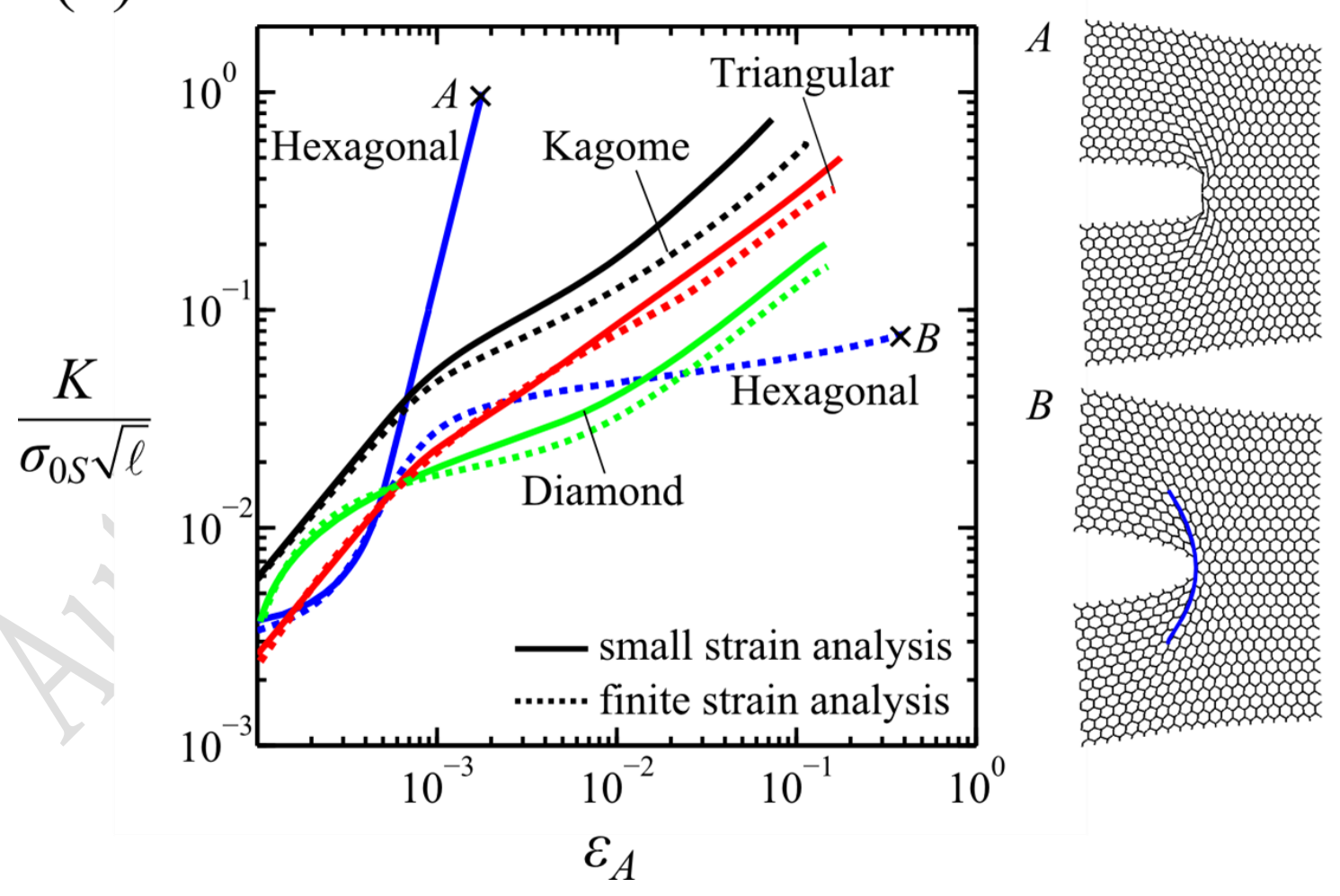




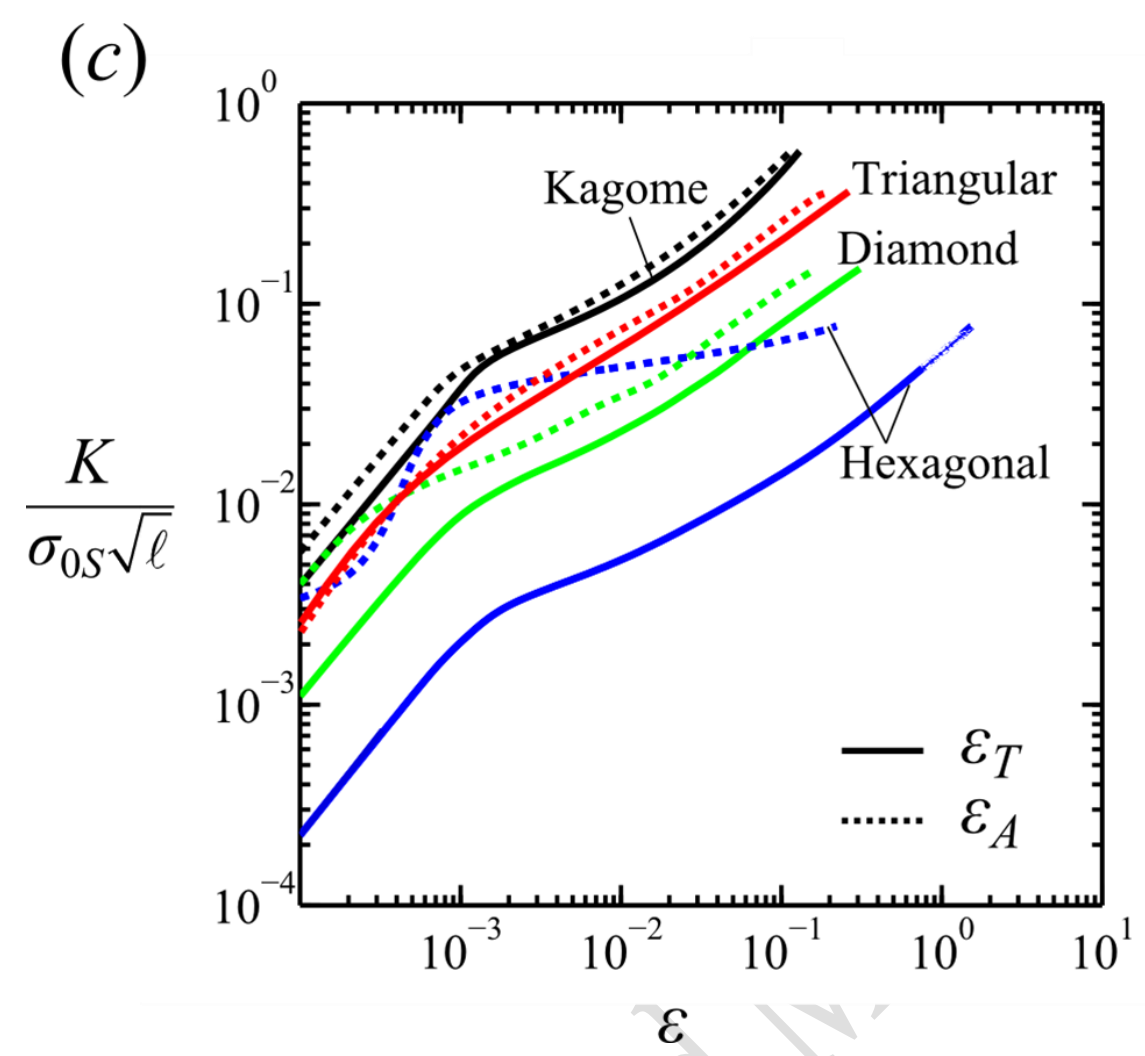

Fig 5. Mode I fracture toughness of the four topologies for $\bar{\rho}=0.05$ and $n=10$ according to (a) local tensile strain (LTS) criterion, (b) average tensile strain (ATS) criterion. (c) A comparison of predictions by the LTS and ATS criteria for the finite strain case. 
(a)

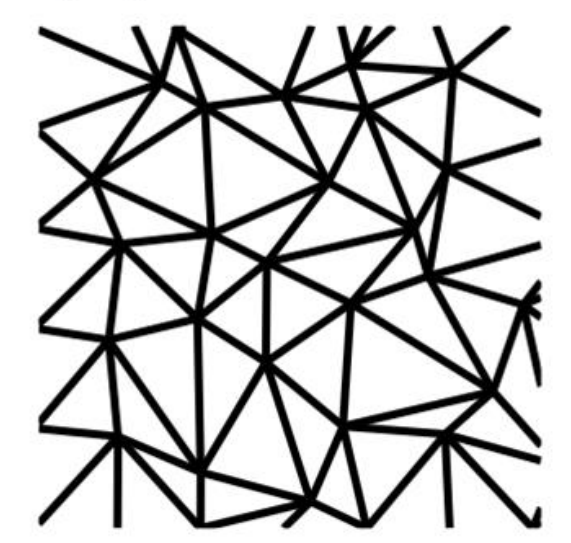

(c)

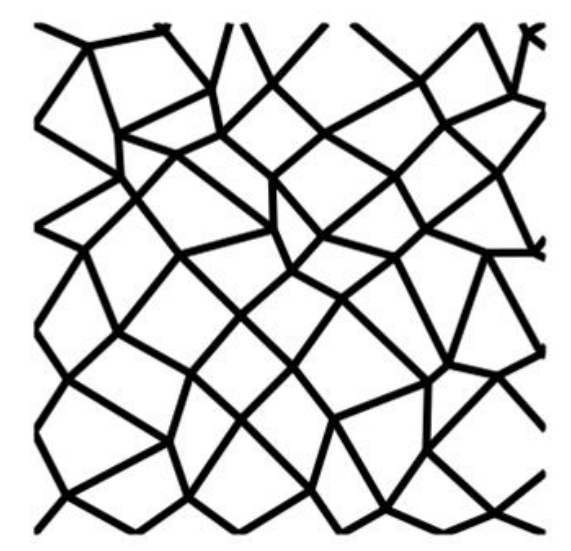

(b)

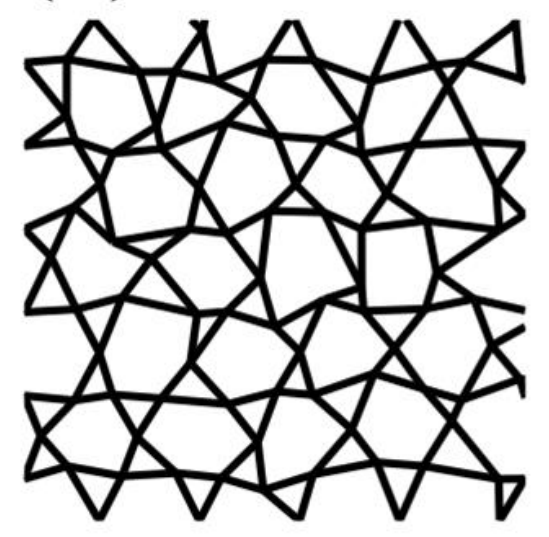

(d)

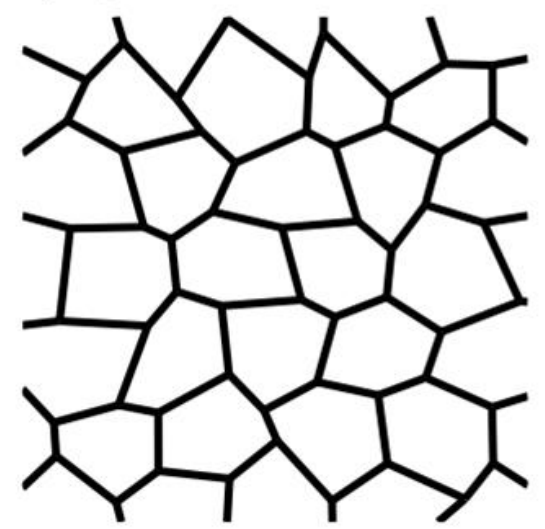

Fig 6. Imperfect lattice topologies $(R / \ell=0.5)$ for $(a)$ triangular lattice, $(b)$ kagome lattice, $(c)$ diamond lattice, $(d)$ hexagonal lattice. 


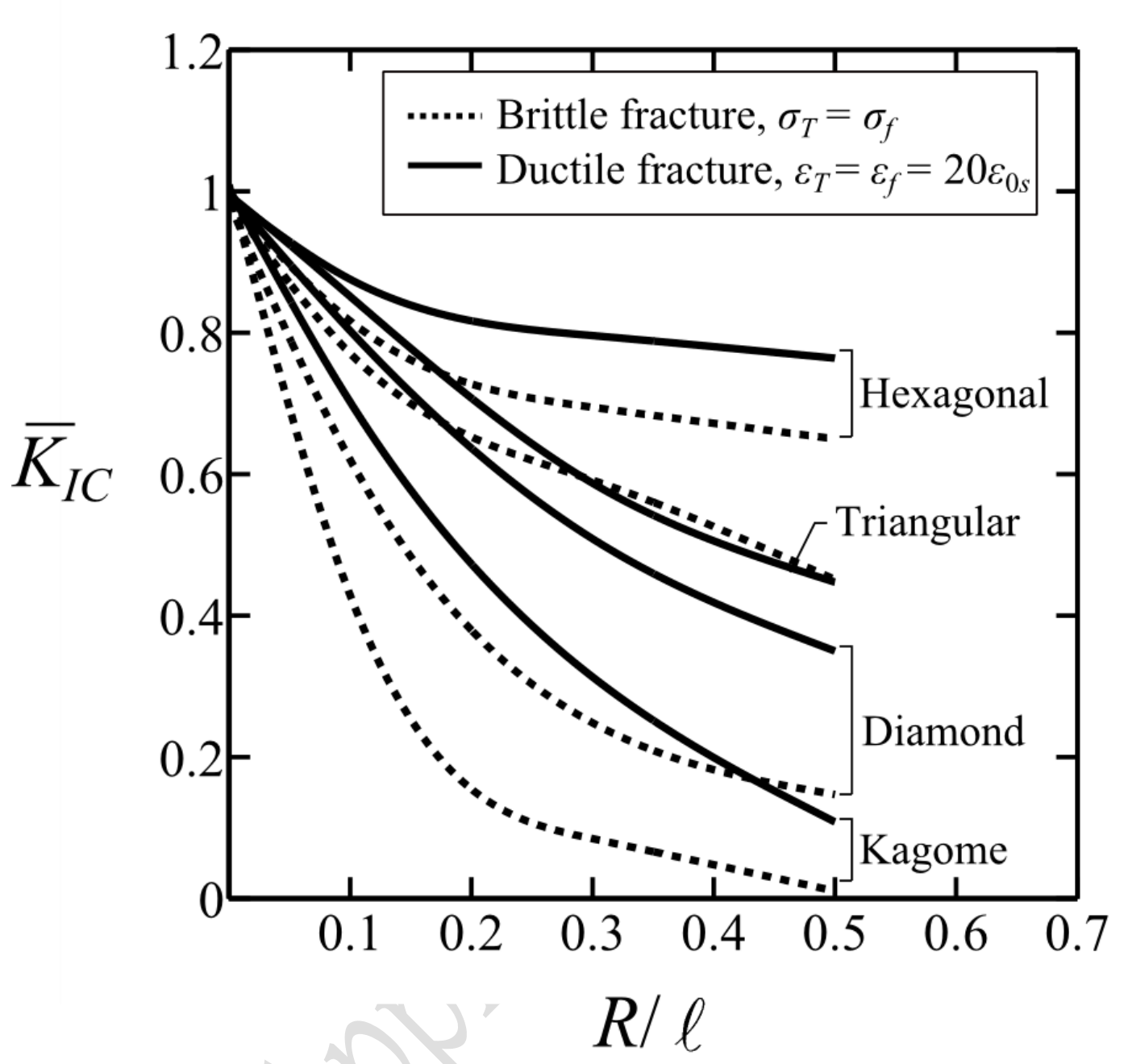

Fig 7. The normalised fracture toughness versus $R / \ell$ of imperfect lattices, for the choice $\bar{\rho}=0.025$ and $n=10$. 


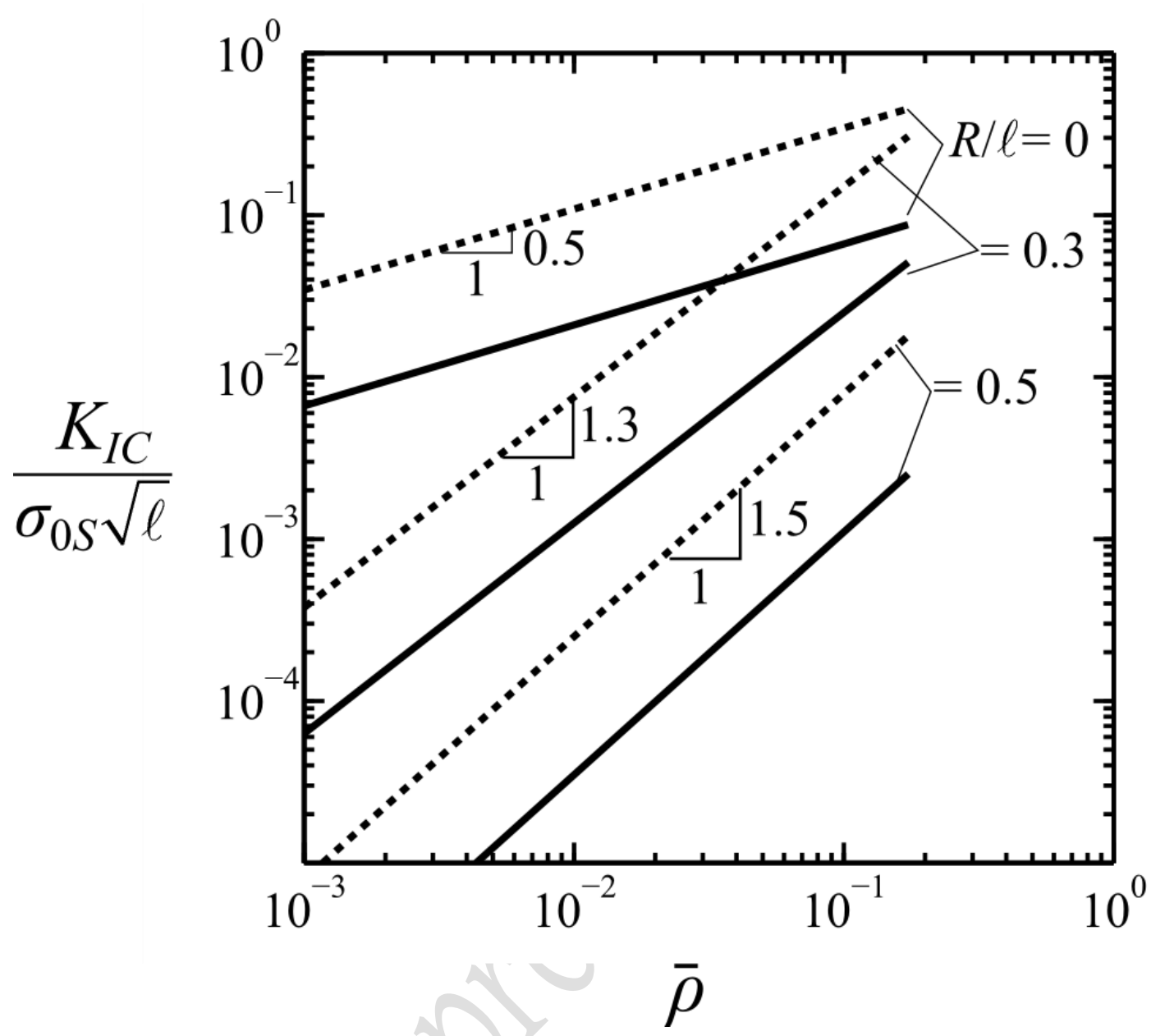

Fig 8. Dependence of fracture toughness of kagome lattice upon relative density $\bar{\rho}$ for the $R / \ell=0,0.3$ and 0.5 . 
(a)

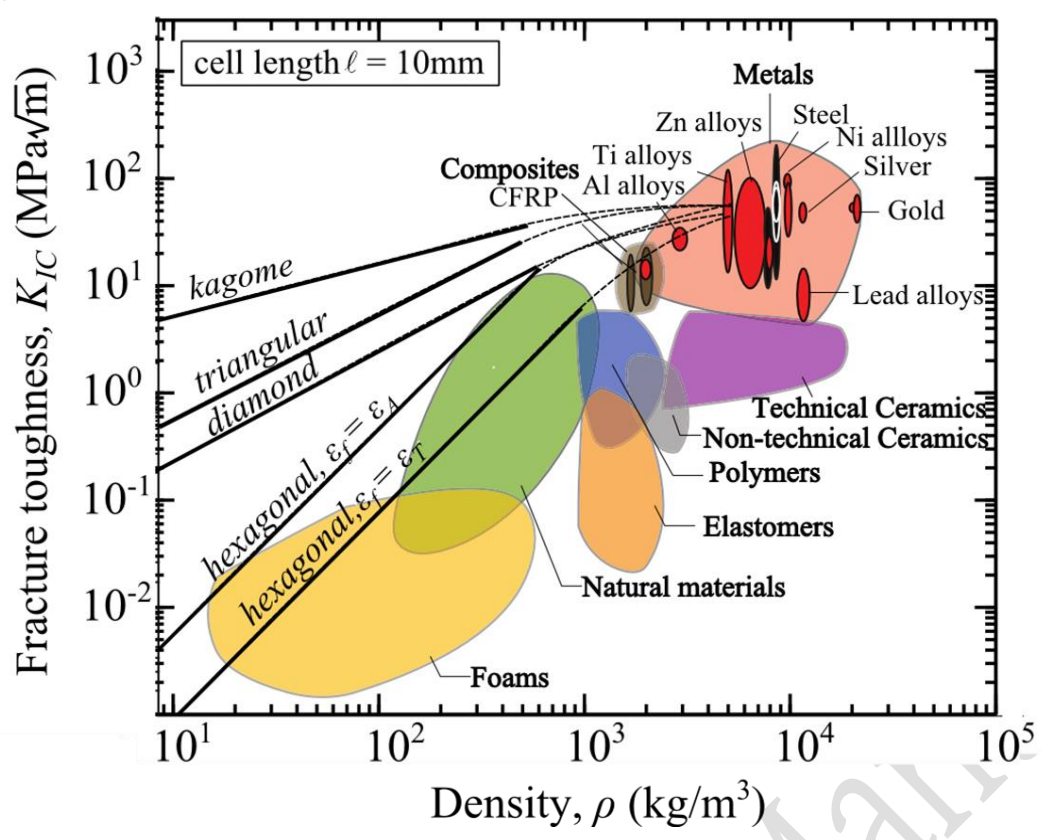

(b)

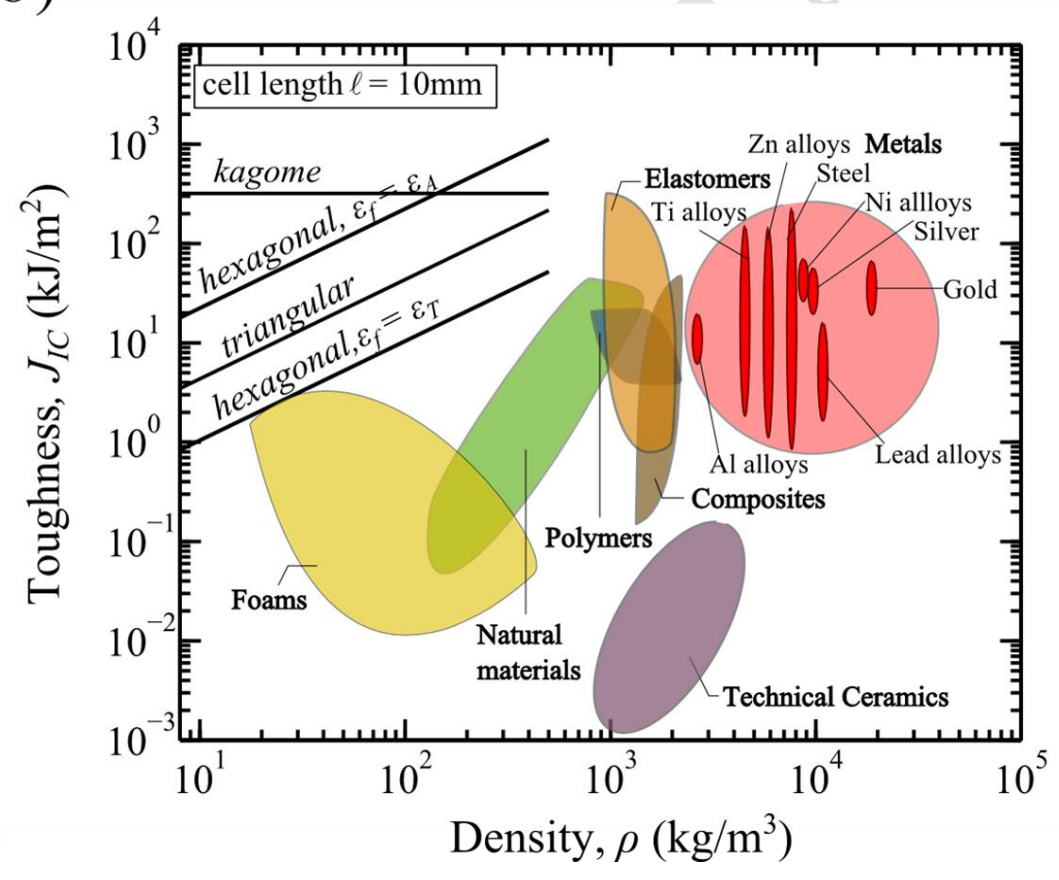

Fig 9. Material property charts (Material Property CES Selector software by Granta Design) for (a) fracture toughness versus density, (b) toughness versus density. Predictions are included for Ti-6Al-4V lattices of cell length $\ell=10 \mathrm{~mm}$ 